\title{
Numerical study of Taylor bubbles rising in a stagnant liquid using a Level-Set / Moving-Mesh method
}

\author{
E. Gutiérrez ${ }^{\mathrm{a}, \mathrm{b}}$, N. Balcázara ${ }^{\mathrm{a}}$, E. Bartrons ${ }^{\mathrm{a}, \mathrm{b}}$, J. Rigola ${ }^{\mathrm{a}}$ \\ ${ }^{a}$ Heat and Mass Transfer Technological Center (CTTC), Universitat Politècnica de Catalunya - BarcelonaTech (UPC) \\ ETSEIAT, Colom 11, 08222 Terrassa (Barcelona), Spain \\ ${ }^{b}$ Termo Fluid S.L., Avda Jacquard 97 1-E, 08222 Terrassa (Barcelona), Spain
}

\begin{abstract}
An Arbitrary Lagrangian-Eulerian formulation has been posed to solve the challenging problem of the threedimensional Taylor bubble, within a Conservative Level Set (CLS) framework. By employing a domain optimization method (i.e. the moving mesh method), smaller domains can be used to simulate rising bubbles, thus saving computational resources. As the method requires the use of open boundaries, a careful treatment of both inflow and outflow boundary conditions has been carried out. The coupled CLS - Moving Mesh method has been verified by means of extensive numerical tests. The challenging problem of the full three-dimensional Taylor bubble has then been thoroughly addressed, providing a detailed description of its features. The study also includes a sensitivity analyses with respect to the initial shape of the bubble, the initial volume of the bubble, the flow regime and the inclination of the channel.

Keywords: Taylor bubble, Arbitrary Lagrangian-Eulerian formulation, Level set method, Open boundary condition, Multiphase flow, Unstructured meshes
\end{abstract}

\section{Introduction}

The slug flow is of fundamental importance in a vast variety of engineering applications and natural phenomena. This flow pattern consists of bullet-shaped bubbles separated by liquid slugs. The bubbles almost completely fill the channel cross section, where at most a thin liquid film separates them from the wall. In reference to its applications, the growing interest in miniaturization of chemical unit operations makes slug flow an important area of study [1. In addition, due to the fact that biomedical studies are becoming increasingly important for the scientific community, slug flows seems to be the key to understand complex blood flow cases, i.e. embolisms. Other relevant scientific fields are also directly related to this flow pattern: microfluidics, volcanology [2], geothermal power plants, gas and oil extraction, cryogenic fluids, etc.

To understand these flows the elemental problem of a single Taylor bubble should be thoroughly comprehended, laying a solid foundation for the analysis of more complex cases. Buoyant bubble problems need the use of large domains to achieve proper capture of the phenomena. That is due to the need of leaving enough vertical space for the bubble to reach its steady state. This introduces a problem: the loss of computational efficiency due to the resolution of areas with no influence in the calculation of the dynamic properties of the bubble. In effect, on a specific point of the simulation, the region of interest includes the bubble and its 
surroundings, and also the zones over which the upwind and downwind disturbances are propagated. This region of interest does not include areas far from the bubble, in which the fluid remains totally quiescent.

To avoid this waste of computational resources, several approaches have been tested. In some cases, improving the efficiency is not only advisable, but also mandatory: for example, when very fine meshes are needed in order to accurately reproduce small scale turbulent motions. The most usual solution is to reduce the computational domain and impose periodic boundary conditions at its limits. However, this may cause the bubble to interact with its own wake if the length of the domain is not large enough, altering its motion. A solution for this problem could be to use a fringe zone, a region above the rising bubble where the velocity field is reinitialized. Several studies have been published using this method [3, 4, 5]. Other approaches can also be adopted by using open boundaries instead of periodic boundaries. The problem can be formulated employing an Arbitrary Lagrangian-Eulerian (ALE) formulation. In this case, since the mesh is moving along the bubble rise, a short computational domain is enough. Moving mesh methods have been broadly used in CFD [6, 7, 8]. It is starting to be applied particularly in bubbles and drops problems [9]. Another similar method which can be used with identical aim and which also need open boundaries is the non-inertial reference frame method, where a new reference frame is attached to the bubble centroid, and the dynamics of the bubble are analysed from this frame. This method has already been used within the context of bubbly flows [10, 11].

As shown above, rising bubbles problems frequently involve the use of open boundaries. A proper design of these boundaries (specially the outflow) could have an overriding impact in the behaviour of the simulation. The outflow boundary should allow the outlet of disturbances, with a minimum influence in the rest of the domain. Different approaches have been adopted to deal with outflow boundary conditions in other rising bubbles studies [12, 13, 10. This work undertakes the task of developing a suitable outflow boundary condition aiming to minimize the global mass error derived from the presence of this boundary. In this regard, a mass correction step is added to the well-known fractional step method [14, 15].

In order to deal with the multiphase domain, several methods are available in the literature. In general, these methods can be classified in two main groups: those using an Eulerian framework and those using a Lagrangian one. The latter is composed of front-tracking methods [16, which precisely describe the multiphase flow, but they are complex to implement due to the need for re-meshing at each iteration. Inside the group of methods that use an Eulerian approach, there are three main types: the volume-of-fluid (VOF) methods [18, the level set (LS) methods [19] and hybrid methods, for example, the VOF/LS method [20]. The VOF methods have the advantage of inherently conserving the mass, but the calculus of the geometrical properties at the interface is troublesome. On the contrary, Level-Set approaches precisely calculate these geometrical properties of the interface (normal and curvature), but they have drawbacks in mass conservation. The hybrid methods usually solve both problems at the expense of increasing the computational cost. The Conservative Level-Set method (CLS) used in the present work was developed by Balcázar et al. [21, in the framework of finite-volume discretization and unstructured meshes. This method greatly reduces the problem of mass conservation of the standard Level-Set methods, and it was thoroughly verified in [22, 23.

In the present paper, the challenging problem of the full three-dimensional Taylor bubble is addressed. Since the publications of the original works of Davies and Taylor [25] and White and Beardmore [26], several approaches have been assessed to simulate Taylor bubbles. Rigorous experimental research have been reported [27, 28, 29, 30], theoretical models have been proved [31, 32] and numerical methods have been addressed, by using Volume of Fluid method [33, Front Tracking method [34, Lattice Bolzmann method [35, 36], and others [37, 38. To the authors' knowledge, the present work is the first approach to the Taylor bubble problem by means of a Conservative Level Set method. Regarding the aforementioned numerical studies, the proposed method solve three typical problems encountered when the Taylor bubble problem is addressed. First, as 
three-dimensional unstructured meshes are employed, 3D circular cross-section tubes can be directly studied, with no need of using a simplified approach (i.e. assuming axisymmetric). Second, by using the CLS method, the mass conservation problem which is known to affect to standard level-set formulations is circumvented. Finally, by using a domain optimization method, the covered domain is small compared with other methods, thus saving computational resources. Therefore, a simulation for some specific conditions has been carried out, allowing comparison against experimental data [29] and other numerical studies [38]. Furthermore, in order to check the validity of the method in a wide variety conditions, other studies have been considered, e.g. sensitivity to the shape of the initial bubble, sensitivity to variations in the initial volume of the bubble, sensitivity to flow regime (comparing the results against those of Quan [34] and Hayashi et al. [39]), and sensitivity to the channel inclination. As to this last topic, there are several valuable studies present in the literature that aims to solve the Taylor bubble problem in inclined channels. For instance, Amaya-Bower and Lee [36] numerically solved the problem in a square cross-section channel, and Shosho and Ryan [30] studied several mixtures at different inclinations.

The work is organized as follows: Sec. 2 presents the mathematical formulation of the level-set multiphase approach (within an ALE framework), and Sec. 3 includes an explanation of the used numerical methods. Thereafter, for the sake of verifying the posed method, a numerical experiment is carried out in Sec. 4 . followed by the solution of the three-dimensional Taylor bubble (Sec. 4.2), providing particular studies and a complete description of its features. Finally, Sec. 5 presents the concluding remarks.

\section{Mathematical formulation}

Assuming incompressible flow, Newtonian fluids, no mass transfer at the interface between fluids, constant surface tension coefficient $\sigma$ and the use of a moving mesh, the Navier-Stokes equations governing the fluid motion are written as [6, 21]:

$$
\begin{gathered}
\frac{\partial}{\partial t}(\rho \mathbf{v})+\nabla \cdot\left(\rho \mathbf{v}\left(\mathbf{v}-\mathbf{v}_{\text {mesh }}\right)\right)=-\nabla p+\nabla \cdot \mu\left(\nabla \mathbf{v}+(\nabla \mathbf{v})^{T}\right)+\rho \mathbf{g}+\sigma \kappa \mathbf{n} \delta_{\Gamma}+\Psi_{\rho_{0}} \\
\nabla \cdot \mathbf{v}=0
\end{gathered}
$$

where $t$ is the time, $\rho$ and $\mu$ are respectively the fluid density and viscosity, $\mathbf{v}$ is the velocity field, $p$ is the pressure field, $\mathbf{g}$ is the gravity acceleration, $\mathbf{n}$ is the unit normal vector to the interface, $\kappa$ is the interface curvature, and $\delta_{\Gamma}$ is the Dirac delta function located at the interface $\Gamma$. $\mathbf{v}_{\text {mesh }}$ is the mesh velocity, which for this case is equal to the vertical component of the bubble velocity (see App. A. $\Psi_{\rho_{0}}=-\rho_{0}$ g represents an extra source term needed to compensate the weight of the fluids within the domain [16, 23, where $\rho_{0}=\frac{1}{V_{\Omega}} \int_{\Omega} \rho \mathrm{d} V$ and $V_{\Omega}$ is the domain's volume. Finally, $\rho$ and $\mu$ can be obtained by the following expressions:

$$
\begin{aligned}
\rho & =\rho_{1} H_{1}+\rho_{2}\left(1-H_{1}\right) \\
\mu & =\mu_{1} H_{1}+\mu_{2}\left(1-H_{1}\right)
\end{aligned}
$$

where subscripts 1 and 2 refer to continuous fluid and bubble fluid respectively, and $H_{1}$ is the Heaviside function, which takes the value of 1 in $\Omega_{1}$ and 0 in $\Omega_{2}$. These $\Omega_{1}$ and $\Omega_{2}$ are the corresponding subdomains associated with the two fluids. At discretized level, physical properties are smoothed according to the CLS method [17, 21, in order to avoid numerical instabilities of the interface.

\subsection{Interface capturing}

The two main difficulties of simulating fluid interfaces are to keep up a sharp interface and to accurately calculate the surface tension [16]. In order to deal with this issues, we use the CLS method for interface 
capturing [21]. In this method, the regularized indicator function $\phi$ is used in order to implicitly represent the interface:

$$
\phi(\mathbf{x}, t)=\frac{1}{2}\left(\tanh \left(\frac{d(\mathbf{x}, t)}{2 \varepsilon}\right)+1\right)
$$

Here, $d(\mathbf{x}, t)=\min _{x_{\Gamma}(t) \in \Gamma}\left(\left|\mathbf{x}, \mathbf{x}_{\Gamma}(t)\right|\right)$ is the signed distance function and $\varepsilon$ is a parameter for controlling the thickness of the interface. This level set function takes the value 0 in one fluid, and 1 in the other. It varies continuously along the interface, which can be located by getting the $\phi=0.5$ isosurface. Based upon this level-set function, the fluid properties are regularized as follows:

$$
\begin{aligned}
& \rho=\rho_{1} \phi+\rho_{2}(1-\phi) \\
& \mu=\mu_{1} \phi+\mu_{2}(1-\phi)
\end{aligned}
$$

The level set function is advected by the velocity field obtained from the solution of the Navier-Stokes equations (Eq. 11 and 22); this yields:

$$
\frac{\partial \phi}{\partial t}+\nabla \cdot \phi\left(\mathbf{v}-\mathbf{v}_{\text {mesh }}\right)=0
$$

Due to numerical diffusion, the thickness of the interface tends to widen. In order to deal with this problem, a reinitialization equation is used to compress the interface [40]:

$$
\frac{\partial \phi}{\partial \tau}+\nabla \cdot \phi(1-\phi) \mathbf{n}_{\tau=0}=\nabla \cdot \varepsilon \nabla \phi
$$

where $\tau$ is the pseudo-time. This equation consists of a compressive term $\phi(1-\phi) \mathbf{n}_{\tau=0}$ which compresses the level-set function along the unit normal vector $\mathbf{n}$, and of a diffusion term $\nabla \cdot \varepsilon \nabla \phi$, that maintains the characteristic thickness of the profile proportional to $\varepsilon=0.5 h^{0.9}$, where $h$ is the grid size [21].

\subsection{Surface tension treatment}

By using the Continuum Surface Force (CSF) method developed by Brackbill et al. 41 two challenging issues can be handled: the computation of the curvature $\kappa$ and the application of the resulting pressure jump to the fluids. Following [41, the singular term $\sigma \kappa \mathbf{n} \delta_{\Gamma}$ is rewritten as a volume force:

$$
\sigma \kappa \mathbf{n} \delta_{\Gamma}=\sigma \kappa(\phi) \nabla \phi
$$

where $\mathbf{n}$ and $\kappa(\phi)$ are given by:

$$
\begin{gathered}
\mathbf{n}=\frac{\nabla \phi}{\|\nabla \phi\|} \\
\kappa(\phi)=-\nabla \cdot \mathbf{n}
\end{gathered}
$$

Here, $\nabla \phi$ is evaluated by means of the least-squares method [21].

\subsection{Moving mesh}

Fig. 1 shows a diagram on how the proposed moving mesh method works in rising bubble problems. The mesh is moving at the vertical velocity of the bubble (see App. A. Thus, the bubble apparently stays vertically stationary inside the mesh domain. A zero-velocity inlet boundary condition is imposed at the top of the domain and an outlet boundary condition at the bottom of the domain. These boundary conditions are particularly explained in Sec. 3. Finally, wall conditions are imposed at the lateral boundaries. 


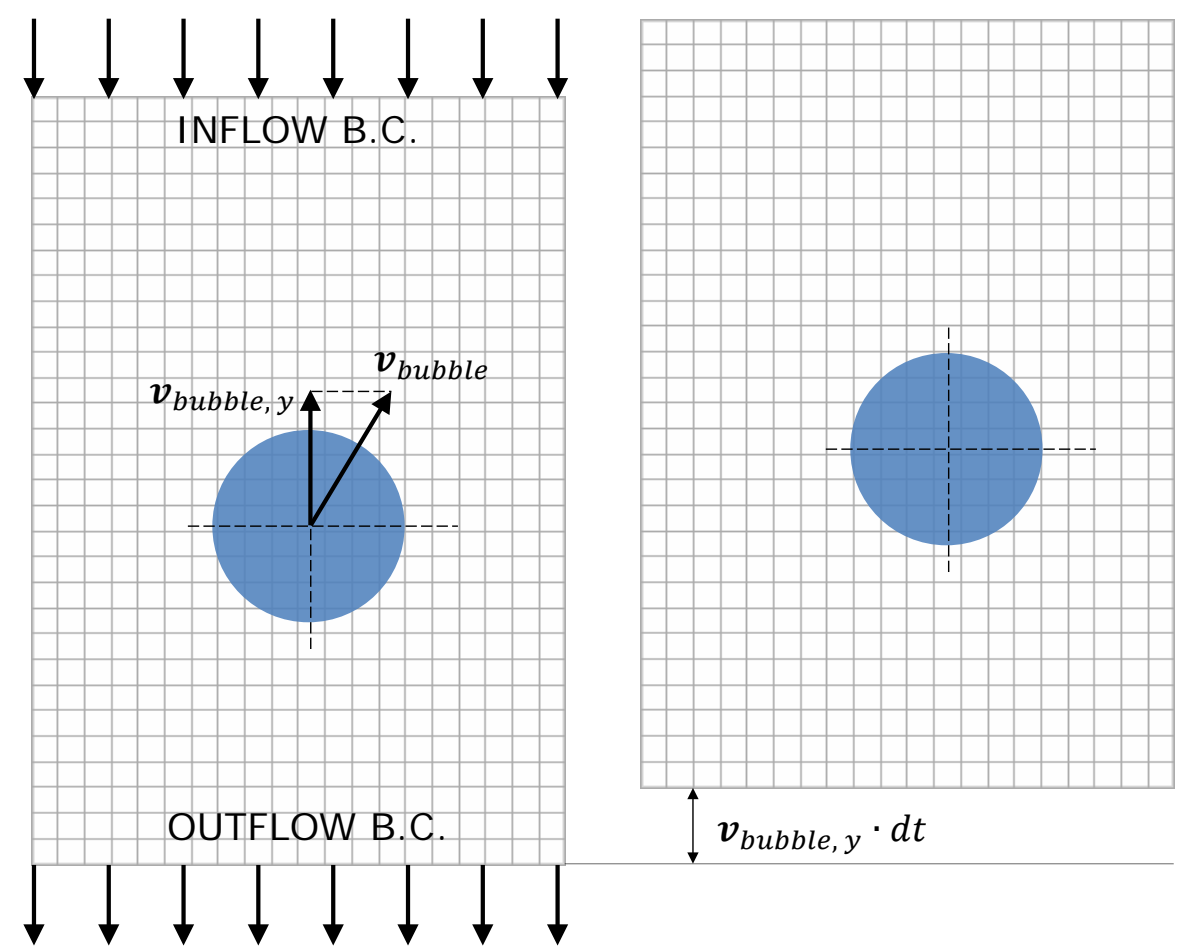

Figure 1: Schematic representation of the moving mesh method, showing two consecutive time instants. The mesh displacement is calculated as the product of the current time step and the bubble vertical velocity.

When an Arbitrary Lagrangian-Eulerian approach is adopted [7, the computational volume should be preserved. This is done by using the so-called Space Conservation Law (SCL) [6]. The mass conservation is procured by enforcing this SCL, which results in the modification of the mass flux through faces, adding the corresponding volume swept in the movement of the face under consideration. As the mesh is trivially moved without deformation at a velocity $\mathbf{v}_{\text {mesh }}$ equal to the bubble's vertical velocity, the calculation of the this correction is straightforward.

\section{Numerical method}

\subsection{Numerical schemes}

The aforementioned equations have been discretized on a collocated unstructured grid arrangment by means of the finite-volume method, according to [21]. A Central Difference (CD) scheme is used to discretize the diffusion terms of the governing equations (Eqs. 1 and 9). A Superbee flux limiter adapted to unstructured meshes [21] is used to discretize the convective term of the advection equation Eq. 8, and a CD scheme is used for the convective term of the momentum equation, Eq. 1. For time discretization, a 3-step-third-order accurate TDV Runge-Kutta scheme [45] is used for advection and reinitialization equations (Eqs. 8 and 9). Finally, CD schemes are used for the compressive and diffusive terms of the reinitialization equation, Eq. 9 20].

The pressure-velocity coupling is solved by means of a Fractional Step method [14, 15, 21, 24]. Momentum 
equation (Eq. 1) is computed in two steps:

$$
\frac{\rho \mathbf{v}^{*}-\rho^{n} \mathbf{v}^{n}}{\Delta t}=\nabla \cdot\left[\rho \mathbf{v}^{n}\left(\mathbf{v}^{n}-\mathbf{v}_{\text {mesh }}^{n}\right)\right]+\nabla \cdot \mu\left(\nabla \mathbf{v}^{n}+\nabla^{T} \mathbf{v}^{n}\right)+\left(\rho-\rho_{0}\right) \mathbf{g}+\sigma \kappa \nabla \phi
$$

For simplicity, this equation is discretized using an explicit Euler scheme, however and explicit AdamsBashforth scheme has been used for computations. The next step of the method is the calculation of the following expression:

$$
\mathbf{v}^{n+1}=\mathbf{v}^{*}-\frac{\Delta t}{\rho} \nabla p^{n+1}
$$

Now, by adding the continuity equation (Eq. 2), the following Poisson equation is obtained to solve the pressure:

$$
\nabla \cdot\left(\frac{1}{\rho} \nabla p^{n+1}\right)=\frac{1}{\Delta t} \nabla \cdot \mathbf{v}^{*}
$$

The discretization of this equation leads to a linear system, which is solved by means of a preconditioned conjugate gradient method. Cell-face velocity is calculated according to [21, 24], in order to avoid pressurevelocity decoupling and to fulfill the incompressible constraint. This cell-face velocity is used to advect the CLS function in Eq. 8 and momentum in Eq. 1.

\subsection{Time step}

A CFL type condition is used to dynamically determine an admissible time step for stable computations. By a straightforward comparison among the terms of Eq. 1. the following global stability condition for the proposed CLS - Moving Mesh method is obtained:

$$
\Delta t=\mathcal{C}_{\mathrm{CFL}} \cdot \min \left(\frac{h}{\left\|\mathbf{v}_{n}\right\|}, \frac{h^{2} \rho_{n}}{\mu_{n}}, \sqrt{\frac{h}{\|\mathbf{g}\|}},\left(\frac{\rho_{1}+\rho_{2}}{4 \pi \sigma}\right)^{1 / 2} h^{3 / 2}, \frac{h}{\left\|\mathbf{v}_{\text {mesh }}\right\|}\right)
$$

where subscript $n$ denotes that the corresponding variable is evaluated at the node $n$ under consideration, $h$ is the characteristic size of the control volume $n$ calculated as the cubic root of the cell volume, and $\mathcal{C}_{\mathrm{CFL}}$ is a safety constant $\left(\mathcal{C}_{\mathrm{CFL}} \approx 0.1\right)$.

\subsection{Inflow boundary condition}

An inflow boundary condition is needed in the upper limit of the domain. This boundary condition aims to achieve a well-defined flow profile at the boundary. The formulation we used is based upon specifying the velocity vector at the corresponding boundary, and using a zero gradient condition for the pressure. These conditions are readily implemented, but the boundary needs to be carefully placed far enough from the bubble. Hence, its interaction with the propagated upstream disturbances is avoided, or at least it is restricted to a negligible effect on the whole flow structure, specially near the bubble. Further details about the placement of this limit are given in subsequent sections.

\subsection{Outflow boundary condition}

As well as the inflow boundary condition, an outflow boundary condition is also required in the proposed method. However, the numerical implementation of the outflow boundary condition is much more burdensome. The real infinite domain is truncated by the outflow boundary, and it would be desirable that this limit is placed sufficiently far in order to avoid interacting with the region of interest. In spite of that, due to practical computational considerations, the boundary is usually placed not so far from the bubble, so a suitable outflow boundary condition is necessary. An incorrect placement of this boundary or an improper 
choice of the boundary condition seriously affects the motion of the bubble. In general, there are three desirable features that an effective outflow boundary condition must have [42]: it should allow the flow to exit the domain with a smooth discharge of disturbances, it should have a minimum effect on the flow near the outlet, and it should have a negligible effect on the bubble motion.

The approach taken in the formulation of the outflow boundary condition was to minimize the global mass error. This is not the only criterion to tackle the task of designing the outlet; other criteria could also be adopted in order to get a suitable boundary condition (e.g. to minimize the local mass error, to minimize the mesh requirements near the boundary, etc.).

The formulation for our outflow boundary condition is based upon a combination of the well-known convective boundary condition (CCB) explained for instance in 43. and the outflow used by Davis and Moore [44. As the fractional step method is going to be used to solve the Navier-Stokes equations [15], a condition for calculating the predictor velocity $\mathbf{v}_{b}^{*}$ in the boundary should be given. In this regard, a convective boundary condition is used:

$$
\frac{\partial \mathbf{v}_{b}^{*}}{\partial t}+v_{c} \frac{\partial \mathbf{v}_{b}^{*}}{\partial n}=0
$$

$n$ indicates the boundary face normal direction and $v_{c}$ is the convective velocity. A characteristic velocity of the problem should be used as $v_{c}$. We assumed $v_{c}=\left\|\mathbf{v}_{\text {bubble }}\right\|$, but other options were also tested (e.g. $v_{c}=U_{T}$, where $U_{T}$ is the expected terminal velocity of the bubble), with negligible differences in the obtained results. Eq. 17 is discretized by using an Euler scheme in time. A correction mass step is carried out at this point, in order to ensure the global mass conservation of the calculated predictor velocity field. The correction mass process differs from others used in the literature and is explained in detail in App. B.

At this point, the pressure at the boundary nodes is set to the corrected hydrodynamic pressure. For our moving mesh method, it yields:

$$
p=\rho \mathbf{g} \cdot \mathbf{x}-\rho_{0} \mathbf{g} \cdot \mathbf{x}
$$

Once the pressure is obtained, the velocity is computed by using Eq. 14

\subsection{Calculation algorithm}

The calculation process needed to advance from the current time step $t^{n}$ to the following one $t^{n+1}$ is detailed in Tab. 1. The present method has been implemented in the context of a parallel c++/MPI code called TermoFluids. The reader is referred to Balcázar et al. 21] for further details on the finite volume discretization of the governing equations. Simulation times of the cases presented in this paper are between 12 and 72 hours (depending mostly on the size of the mesh and the obtained CFL condition). Those cases were run using 32 up to 256 CPUs.

\section{Numerical results and discussion}

\subsection{Validation on single bubble rising in viscous liquid}

In the present section, the proposed CLS - Moving Mesh method is assessed in a three-dimensional rising bubble. Results are compared against those of Balcázar [23].

Flow regime is defined by setting the following dimensionless numbers:

$$
\eta_{\rho}=\frac{\rho_{1}}{\rho_{2}} \quad, \quad \eta_{\mu}=\frac{\mu_{1}}{\mu_{2}} \quad, \quad E o=\frac{g d^{2}\left(\rho_{1}-\rho_{2}\right)}{\sigma} \quad, \quad M o=\frac{g \mu_{1}^{4}\left(\rho_{1}-\rho_{2}\right)}{\rho_{1}^{2} \sigma^{3}}
$$




\begin{tabular}{l}
\hline CLS - Moving Mesh method \\
\hline 1.- Calculate the mesh velocity, as explained in Sec. 2 \\
2.- Choose a suitable time step, as explained in Sec. 3.2 \\
3.- Advect the level set function $\phi$ by solving Eq. 8. \\
4.- Compress the interfaces between both fluids by solving Eq. 9 . \\
5.- Update the density, viscosity, curvature and normal fields. \\
6.- Solve Eq. 1 and 2 by using the fractional step method: \\
- Calculate the predictor velocity. \\
- Perform the mass conservation step explained in App. B \\
- Solve the Poisson equation Eq. 15 to get the pressure field. \\
- Calculate the cell-face velocity $[21]$. \\
7.- Move the mesh. \\
8.- Update mass flow by imposing the SCL. \\
9.- Repeat the previous steps up to the desired time.
\end{tabular}

Table 1: Calculation procedure for the proposed CLS - Moving Mesh method.

$\eta_{\rho}$ and $\eta_{\mu}$ are the density and viscosity ratios, respectively. Eo is the Eötvös number and $M o$ is the Morton number. In the present problem, $\eta_{\rho}=100, \eta_{\mu}=100, E o=39.4$ and $M o=0.065$.

The initial state and the mesh configuration is sketched in Fig. 2 A cylindrical shaped domain is used. We employed the semi-empirical expression obtained by Harmathy [46] to calculate the diameter of the tube $D$ :

$$
\frac{U_{T}}{U_{T}^{\infty}} \simeq 1-\left(\frac{d}{D}\right)^{2}
$$

where $U_{T}^{\infty}$ represents the terminal velocity in an infinite domain, $U_{T}$ is the actual terminal velocity and $d$ is the bubble diameter. Based on this equation, the diameter of the domain $D$ is set to $8 d$ as it leads to accurate enough results, with an error of $1.5 \%$ approximately.

Boundary conditions at inlet and outlet are explained in detail in Sec. 3, and no slip boundary condition is applied at lateral walls. On the one hand, the bubble is initially placed on the cylinder axis at a distance from the outlet $h_{o}$ of $4 d$. On the other hand, distance to inlet $h_{i}$ is $2 d$, giving rise to a total axial distance of $7 d$. The mesh structure is generated by extruding a non-uniform two-dimensional mesh along the cylinder axis. An exponential growth in radial direction is used, and an orthogonal structured grid is fixed in the immediate area of the axis, with a cell size of $h$. A mesh independence study has been carried out. In this study, the minimum control volume size $h$ is set by dividing the bubble diameter into 20,25 and 30 control volumes. The mesh configuration shown in Fig. $2 \mathrm{~b}$ is suitable for this flow regime since the lateral motion of the bubble is practically worthless.

Fig. 3 shows the results corresponding to the test case. Good agreement was found with results reported by Balcázar 23. Finally, Tab. 2 summarizes the obtained results.

\subsection{Validation on Taylor bubble rising in viscous liquid}

In this section, the Taylor bubble problem is studied by using the coupled CLS - moving mesh method. The chosen reference case is the one published by Bugg and Saad 29. (experiment) and by Ndinisa et al. [38. (numerical simulation). Some other cases were also studied in subsequent sections, in order to cover all the phenomena present in the problem (i.e. the ones presented in the papers of Quan [34], Hayashi et al. [39] and Shosho and Ryan [30). 


\begin{tabular}{lcc}
\hline Results & $U_{T}(d g)^{-1 / 2}$ & $\zeta_{3 D}$ \\
\hline CLS - Moving Mesh method $(h=d / 20)$ & 0.595 & 0.7923 \\
CLS - Moving Mesh method $(h=d / 25)$ & 0.604 & 0.7848 \\
CLS - Moving Mesh method $(h=d / 30)$ & 0.607 & 0.7803 \\
Balcázar [23] (fixed mesh) & 0.610 & 0.784 \\
Hnat and Buckmaster [4] (experimental) & 0.622 & - \\
\hline
\end{tabular}

Table 2: Summary of achieved results at dimensionless time $t^{*}=10$ for the three-dimensional rising bubble problem. Dimensionless terminal velocity $U_{T}(d g)^{-1 / 2}$ and sphericity $\zeta_{3 D}$ are shown for the proposed CLS - Moving Mesh method, in comparison with reference data reported by [23] $\left(\eta_{\rho}=100, \eta_{\mu}=6.67 \cdot 10^{3}\right)$, and by [47] $\left(\eta_{\rho}=714, \eta_{\mu}=6.67 \cdot 10^{3}\right)$.

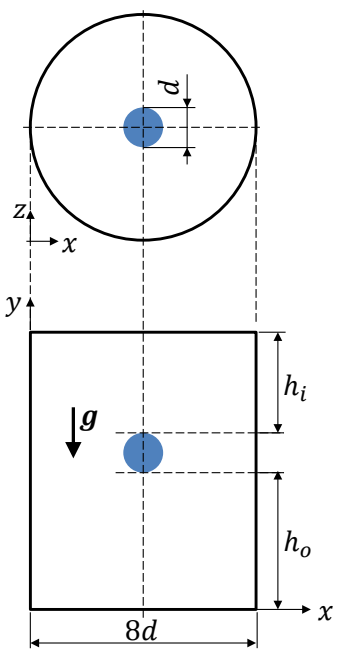

(a)

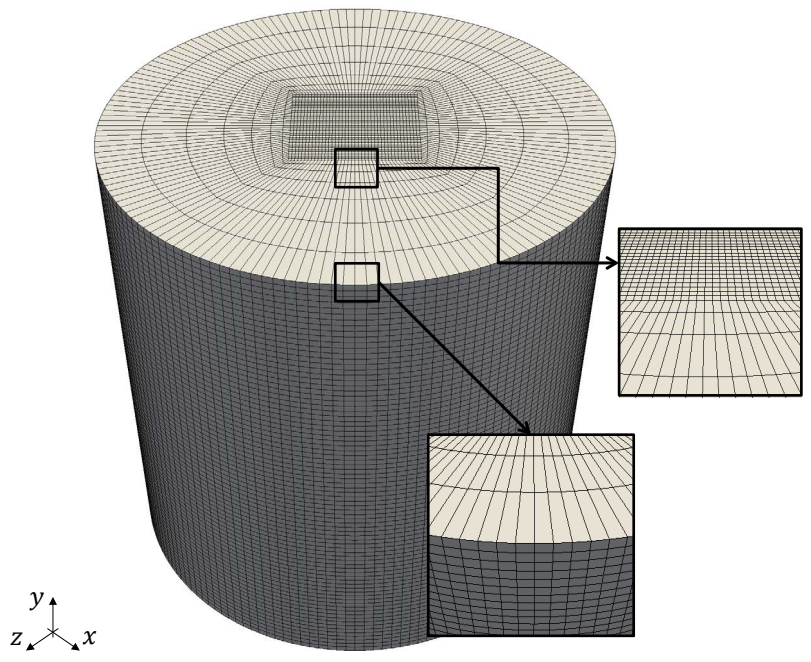

(b)

Figure 2: Representation of (a) computational set-up and (b) mesh configuration of the three-dimensional rising bubble problem, in which $d$ denotes the diameter of the bubble, and $h_{i}$ and $h_{o}$ are the distances from the bubble to the inlet and outlet boundaries, respectively.

All these works have some common defining parameters that set up the problem configuration and, in particular, its initial condition. For example, the size of the bubble is characterized by a dimensionless bubble size parameter $k=2 a / D$, where $a$ is the radius of an equivalent spherical bubble of the same volume, and $D=2 R$ is the diameter of the tube. Then, $a=\left(3 V_{\Omega_{2}} / 4 \pi\right)^{1 / 3}$, where $V_{\Omega_{2}}$ is the volume of the bubble. The initial radius of the bubble $r$ is chosen to be close to the one that will have the bubble in the steady state. In order to do so, the following expression is used, as derivation of the one obtained by Brown [48 when $D \sim r$ :

$$
r=\frac{D}{2}-\sqrt[3]{\frac{3 \mu_{1}^{2}}{4 g \rho_{1}^{2}} \cdot R e_{T}}=\frac{D}{2}-\sqrt[3]{\frac{3 \mu_{1} D^{3 / 2}}{4 g^{1 / 2} \rho_{1}} \cdot F r_{T}}
$$

where the expected terminal Reynolds number $R e_{T}=\rho_{1} U_{T} D / \mu_{1}$ or the expected terminal Froude number $F r_{T}=U_{T} / \sqrt{D g}$ is obtained from the well-known predictions of White and Beardmore 26].

Furthermore, as the used method leaves the bubble quiescent at its starting vertical position, initial distances from the bubble nose to the inlet $h_{i}$ and from the bubble rear end to the outlet $h_{o}$ should be fixed, as they will remain approximately constant during the simulation. On the one hand, the distance $h_{i}$ is set to $D$, since for not too slow regimes $\left(R e_{T}>1\right)$ velocity field perturbations do not propagate beyond $D$ from 


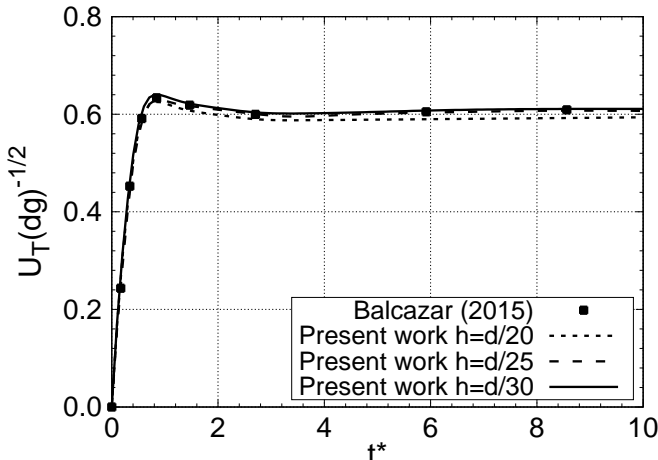

(a)

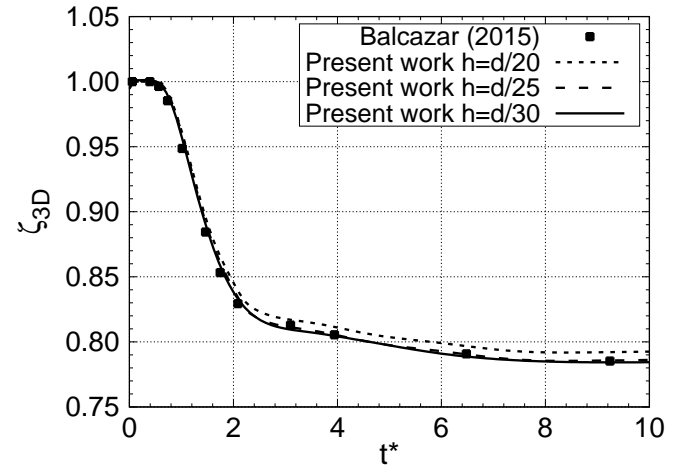

(c)

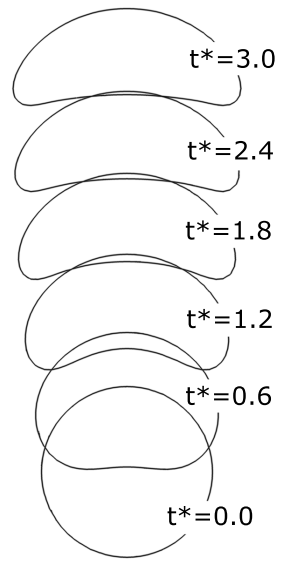

(b)

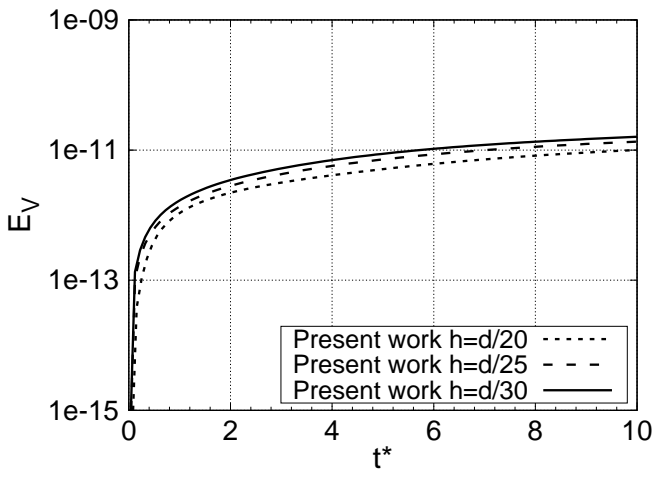

(d)

Figure 3: Three-dimensional standard rising bubble results for the tested method. The graphs show (a) dimensionless terminal velocity $U_{T}(d g)^{-1 / 2}$, (b) bubble shape evolution, (c) sphericity $\zeta_{3 D}$ and (d) global volume error $E_{V}$ over dimensionless time $t^{*}=t g^{1 / 2} d^{-1 / 2}$, for the different mesh resolutions tested.

the bubble nose. On the other hand, the following expression was found useful to determine the distance $h_{o}$ :

$$
h_{o}= \begin{cases}\left(\mathcal{C}_{h_{o}}-1\right) D & \text { if } \operatorname{Re}_{T}<10 \\ {\left[\mathcal{C}_{h_{o}} \log _{10}\left(R e_{T}\right)-1\right] D} & \text { if } \operatorname{Re}_{T}>10\end{cases}
$$

where $\mathcal{C}_{h_{o}}$ is a safety constant $\left(\mathcal{C}_{h_{o}} \approx 2.0\right)$. It is important to note that this equation was successfully used in the context of the method presented in this work. The extrapolation of its applicability to other conditions should be analyzed particularly.

Fig. 4 depicts the initial configuration for the reference case. The simulation is based on the conditions described by Ndinisa et al. [38. The density of the continuous phase $\rho_{1}$ is set to $911 \mathrm{~kg} / \mathrm{m}^{3}$, and its viscosity $\mu_{1}$ is $0.084 \mathrm{~Pa} \cdot \mathrm{s}$. On the other hand, the properties of the bubble's fluid correspond to air at $20^{\circ} \mathrm{C}$. Therefore, $\rho_{2}=1.205 \mathrm{~kg} / \mathrm{m}^{3}$ and $\mu_{2}=1.827 \cdot 10^{-5} \mathrm{~Pa} \cdot \mathrm{s}$. The surface tension coefficient $\sigma$ is set to $0.0328 \mathrm{~N} / \mathrm{m}$. The diameter of the pipe $D$ is set to $0.019 \mathrm{~m}$. Those properties and geometrical parameters yield the following dimensionless groups: $\eta_{\rho}=\rho_{1} / \rho_{2}=756.017, \eta_{\mu}=\mu_{1} / \mu_{2}=4597.701, E o=\rho_{1} g D^{2} / \sigma=100$ and $M o=g \mu_{1}^{4} /\left(\rho_{1} \sigma^{3}\right)=0.015$. For its part, the expected terminal Reynolds number $\operatorname{Re}_{T}=\rho_{1} U_{T} D / \mu_{1}$ is about 27, where $U_{T}$ is the terminal velocity calculated as explained in App. A. No slip boundary condition is 


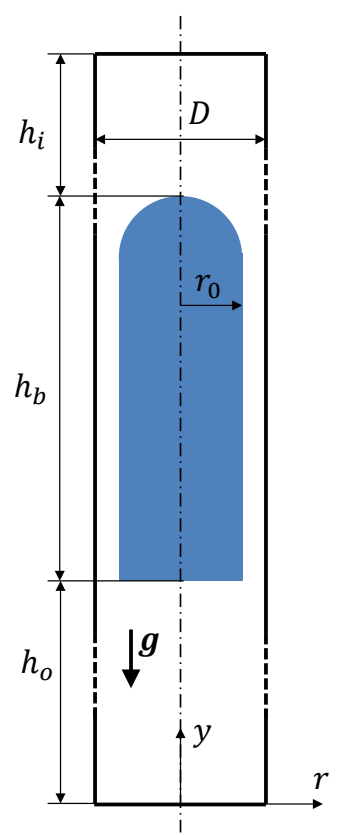

Figure 4: Outline of the initial set-up of the Taylor bubble problem. Here, $r=0.007 \mathrm{~m}$ is the the bubble's radius and $h_{b}=0.0523 \mathrm{~m}$ is its length.

used at the lateral side of the domain. The boundary conditions at inlet and outlet were explained in detail in Sec. 3.

The initial shape of the bubble is a cylinder with an hemisphere at the front end (see Fig. 4). The radius $r$ of this cylinder is $0.007 \mathrm{~m}$ (see Eq. 21). The total axial distance $h_{b}$ of the initial bubble is $0.0523 \mathrm{~m}$, which leads to a total initial volume of $7.697 \cdot 10^{-6} \mathrm{~m}^{3}$, and a parameter $k$ of 1.29 .

The simulation domain is cylindrical and it was meshed using hexahedral control volumes uniformly distributed over the whole space. The meshes were generated by a constant step extrusion of a two-dimensional unstructured grid along the axis of the cylinder, being the step size $y_{\text {length }} / N_{\text {planes }}$, where $y_{\text {length }}$ is the axial length and $N_{\text {planes }}$ is the number of planes in which the vertical axis is divided. Tab. 3 shows a description of the used grids. Three meshes with different resolution were considered, namely $M 1, M 2$ and $M 3$. The mesh resolution was set by dividing the tube diameter $D$ into 52, 76 and 95 control volumes, respectively.

Furthermore, as pointed out above, initial distance form the bubble nose to the inlet $h_{i}$ was set to $D$, and distance from the bubble rear end to the outlet $h_{o}$ was computed by means of Eq. 22, For the conditions of the experiment of Ndinisa et al. 38, we obtained $h_{o} \approx 2 D$. Taking into the account that the axial dimension of the initial bubble $h_{b}$ is $2.75 D$, a total axial distance $y_{\text {length }}$ of $5.75 D$ was found enough to reproduce the ascent of the Taylor bubble until it reached the steady state. This greatly reduces the domain requirements compared to previous works, where axial distances between $8 D$ and $16 D$ are typically used 34. Furthermore, complex meshes could be used, with a relatively large characteristic cell size, and having enough nodes concentration in the important regions of the problem (i.e. the wall vicinities and the wake zone). However, for the sake of simplicity, current simulations were run with quasi-homogeneous meshes.

Fig. 5a shows the evolution of the Reynolds number $R e$ along dimensionless time $t^{*}$. Results are summarized in Tab. 4 where a comparison against other works are presented. Good agreement was found when $M 2$ and $M 3$ meshes are used. With $M 1$ mesh, the final velocity is less accurate. Furthermore, Fig. 5b depicts 


\begin{tabular}{cccccc}
\hline $\begin{array}{c}\text { Mesh } \\
\text { name }\end{array}$ & Mesh size & $\begin{array}{c}\text { Cells per } \\
\text { plane }\end{array}$ & $N_{\text {planes }}$ & $y_{\text {length }}$ & $h$ \\
\hline$M 1$ & $5.3 \cdot 10^{5}$ & 2098 & 254 & $5 D$ & $D / 52$ \\
$M 2$ & $1.9 \cdot 10^{6}$ & 5043 & 380 & $5 D$ & $D / 76$ \\
$M 3$ & $3.7 \cdot 10^{6}$ & 7891 & 475 & $5 D$ & $D / 95$ \\
\hline
\end{tabular}

Table 3: Description of the meshes used in the Taylor bubble problem, where $N_{\text {planes }}$ is the number of planes in which the vertical axis is divided and $y_{\text {length }}$ is the length of this vertical axis.

the bubble profile evolution along the dimensionless time $t^{*}$.

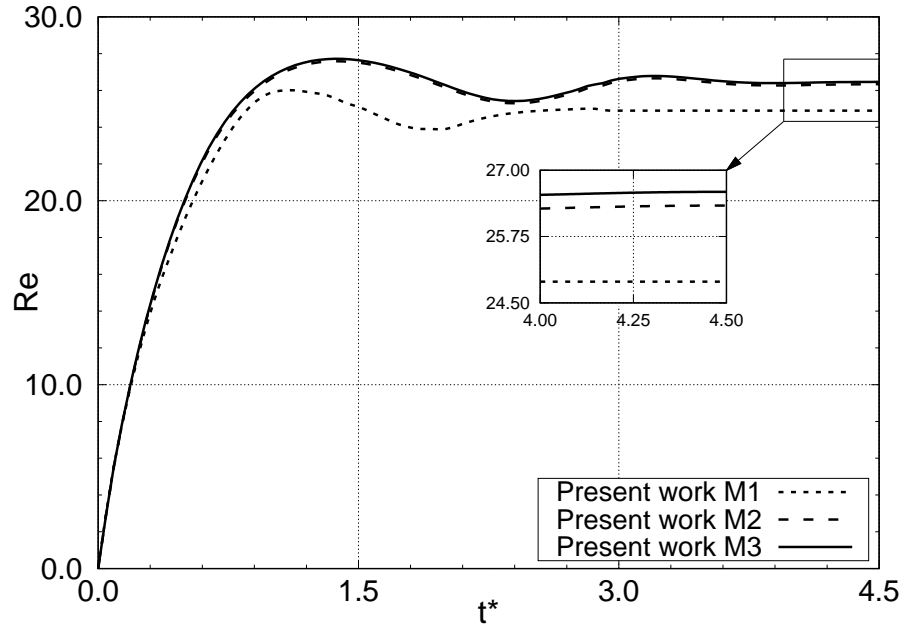

(a)

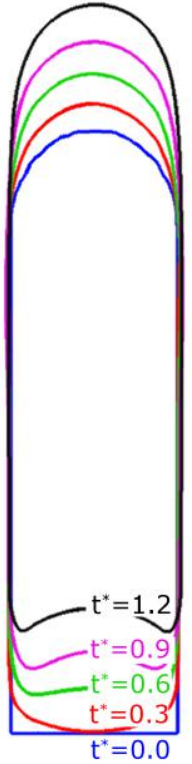

(b)

Figure 5: Reynolds number evolution and profiles evolution of the tested Taylor bubble. The bubble profile after $t^{*}=1.2$ remains approximately constant.

\begin{tabular}{ccc}
\hline Case & $U_{T}$ & $E_{U_{T}}$ \\
\hline Present work $(M 1)$ & $0.1210 \mathrm{~m} / \mathrm{s}$ & $7.63 \%$ \\
Present work $(M 2)$ & $0.1277 \mathrm{~m} / \mathrm{s}$ & $2.52 \%$ \\
Present work $(M 3)$ & $0.1286 \mathrm{~m} / \mathrm{s}$ & $1.83 \%$ \\
Ndinisa et al. [38] & $0.140 \mathrm{~m} / \mathrm{s}$ & $6.87 \%$ \\
White and Beardmore [26] & $0.1272 \mathrm{~m} / \mathrm{s}$ & $2.90 \%$ \\
Bugg and Saad [29] & $0.131 \mathrm{~m} / \mathrm{s}$ & - \\
\hline
\end{tabular}

Table 4: Summary of achieved results at dimensionless time $t^{*}=4.5$ for the three-dimensional Taylor bubble problem, where $E_{U_{T}}$ is the relative error compared to the experimental results of Bugg and Saad [29]. 


\subsubsection{Results discussion}

Fig. 6 shows several velocity profiles plotted over different sections by comparing them with the reference data. Results are shown for the three studied meshes. On the other hand, Fig. 7 sketches the velocity field and the streamlines. By analysing these images, a fairly accurate picture of the velocity field can be depicted.

First, the normalized axial velocity along the tube axis above the bubble nose is plotted in Fig. 6a. Results of this graph are referred to an auxiliary reference frame located in the bubble nose. Ahead of the bubble, the suspending fluid is perturbed by the ascent of the bubble. However, as shown in Fig. 6a, the bubble does not have a strong influence on the fluid above it, since the axial velocity tends toward zero at a distance of $D / 3$. Close to the bubble nose, the fluid is strongly radial, since the bubble is moving upwards and the fluid ahead of it is pushed sideways. This can be clearly seen in Figs. 6b and 6c where the normalized axial and radial velocities across the tube radius at $0.111 D$ above the bubble nose are plotted, respectively. Figs. 6d and 6e depict the normalized axial and radial velocities in the developing film at $0.504 D$ below the bubble nose, showing that, as we descend into the developing film, a strong radial velocity component is still observed, specially close to the fluids' interface. The developing film speeds up and thins as it falls, until the shear stress at the wall is capable of withstanding the weight of the film. The fully developed film is then formed, giving rise to an essentially axial and constant flow. When the rear end is achieved, the axial velocity is dramatically reduced (see Fig. 6f, showing the normalized axial velocity in the wake of the bubble at $0.2 D$ below the bubble). At this point the flow becomes strongly radial, since the fluid from the wall is transferred toward the cylinder axis. The radial velocity component swiftly decays to zero near the tube axis, where the suspending fluid is moving upward with a similar velocity as the bubble's one.

The present results are confirmed by experimental and numerical studies reported by Bugg and Saad [29] and Ndinisa et al. [38. For meshes $M 2$ and $M 3$, good agreement can be found in all of the results. On the other hand, mesh $M 1$ does not seem fine enough to correctly replicate these results. For the results obtained with $M 2$ and $M 3$ meshes, the slight disagreements can be explained based on the ambiguity of locating an specific section relative to the bubble nose. In effect, results in Fig. 6 show notable changes when visualizing sections near each other, highlighting the importance of using fine meshes. This observation is consistent with Ndinisa et al. work [38.

The sketches of the velocity field and the streamlines (Fig. 7) agree qualitatively with results reported by Bugg and Saad [29] and by Ndinisa et al. [38]. These images underscore the essentially axisymmetric nature of this problem. Furthermore, a large vortex is observed inside the Taylor bubble. Due to the selected flow regime, no vortex appears in the closed-wake of the bubble.

\subsection{Effect of the initial shape of the Taylor bubble}

Besides the initial shape proposed above, others were also tested in order to check the influence of the initial condition on the obtained results, with the same dimensionless numbers than in the previous section. The different initial bubble forms tested are: a cylinder with an hemisphere at the front end, a cylinder with two hemispheres, a standard cylinder, a cylinder with an hemisphere at the front end and a notched rear end, and a shape close to the stationary. This last form was obtained by taking the bubble profile from a previous simulation. Fig. 8a depicts these different initial bubble shapes. All of them have the same total volume of $7.697 \cdot 10^{-6} \mathrm{~m}^{3}(k=1.29)$, and the same radius $r$. The radius of the notch in the respective case is $0.8 r$. Fig. $8 \mathrm{~b}$ shows the time evolution of these distinct cases. The initial bubble shape only has influence in the transient period and in the total time needed to achieve the final state. In this regard, the shape close to the stationary is narrowly the first to achieve the stationary state, at approximately $t^{*}=3.0$. The governing factor in the time evolution of the different shapes seems to be the shape of the bubble's frontal area (at least for the selected parameter $k$ ). While all the shapes with an hemisphere in the front part of the bubble 


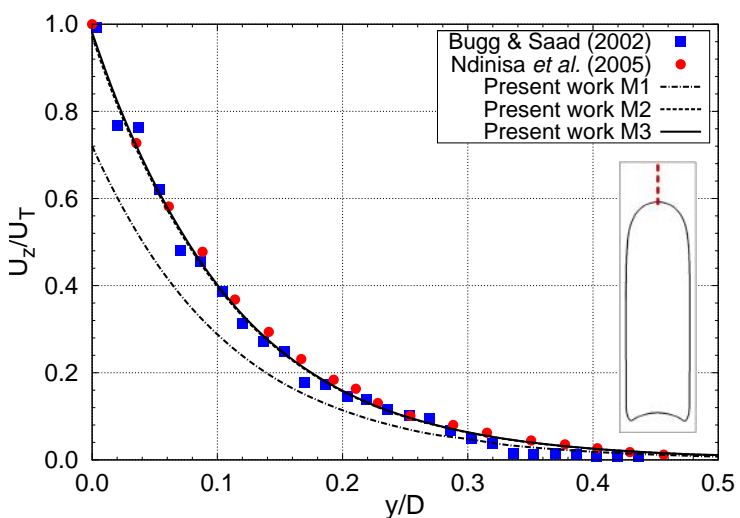

(a)

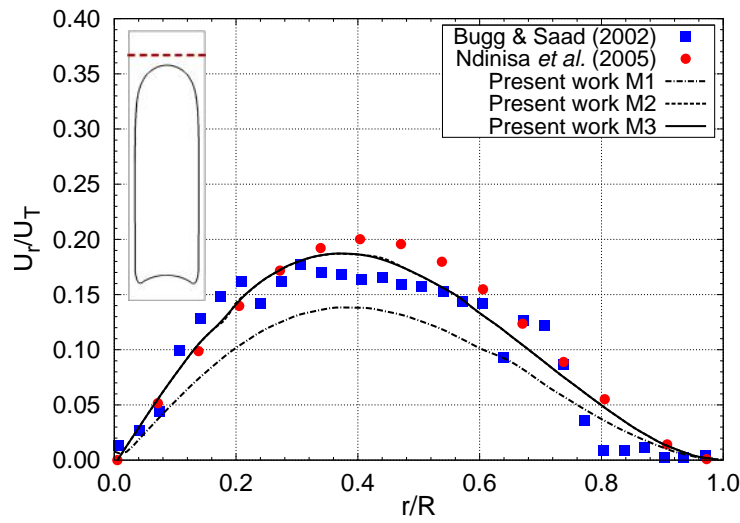

(c)

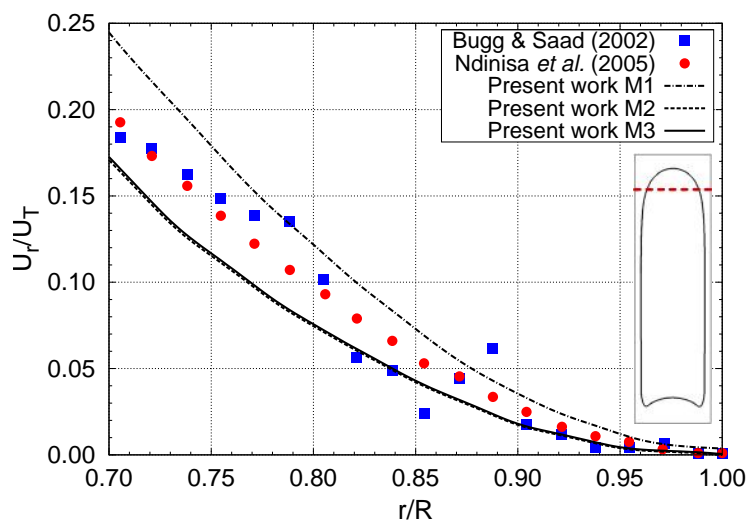

(e)

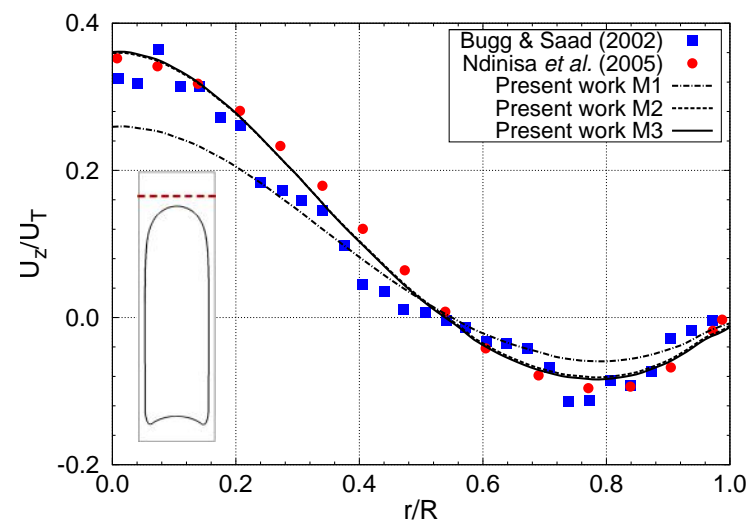

(b)

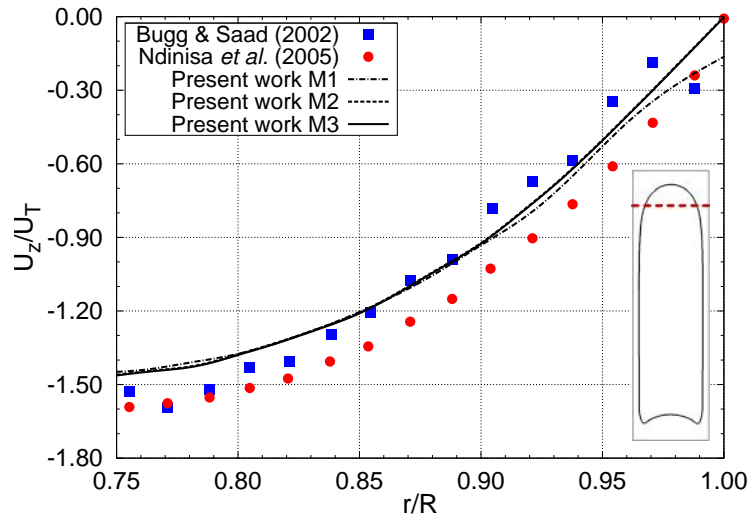

(d)

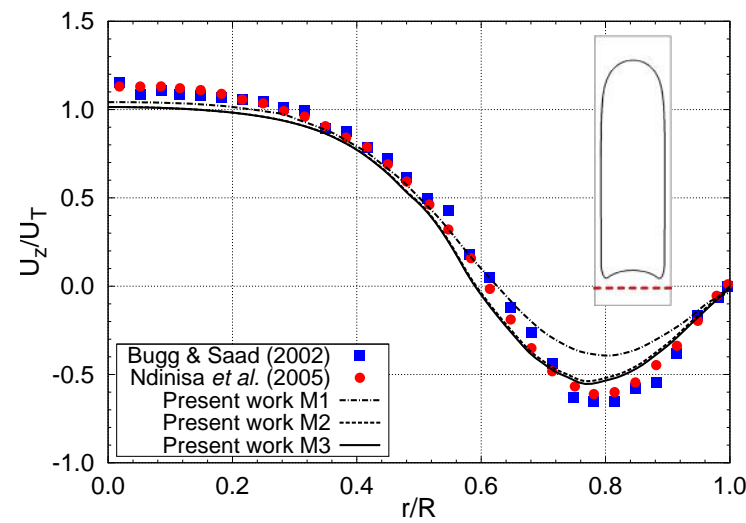

(f)

Figure 6: Results from the three-dimensional Taylor bubble problem, showing (a) the normalized axial velocity in the tube axis above the bubble nose, (b) the normalized axial velocity in a section above the bubble, (c) the normalized radial velocity in a section above the bubble, $(\mathrm{d})$ the normalized axial velocity in the developing film, (e) the normalized radial velocity in the developing film and (f) the normalized axial velocity in the wake of the bubble. 


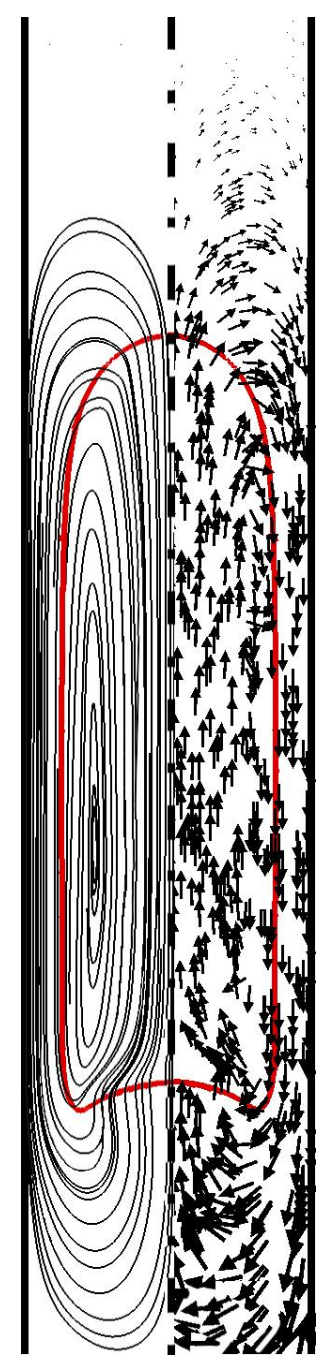

(a)

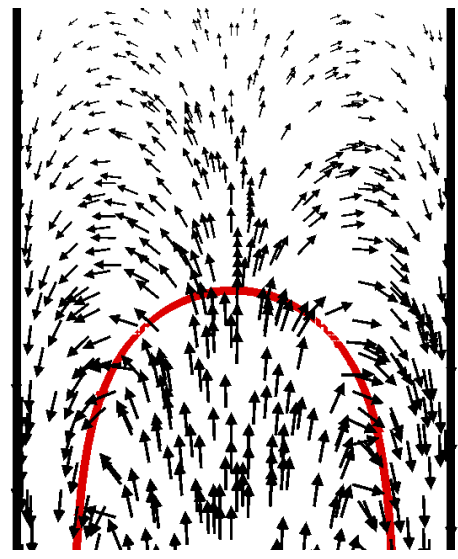

(b)

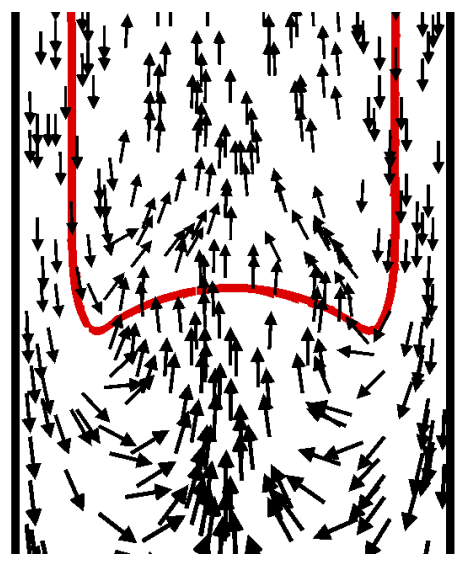

(c)

Figure 7: Streamlines and velocity field details of the three-dimensional Taylor bubble problem.

evolve similarly, the standard cylinder presents a very different time evolution, as well as the shape close to the stationary state. 


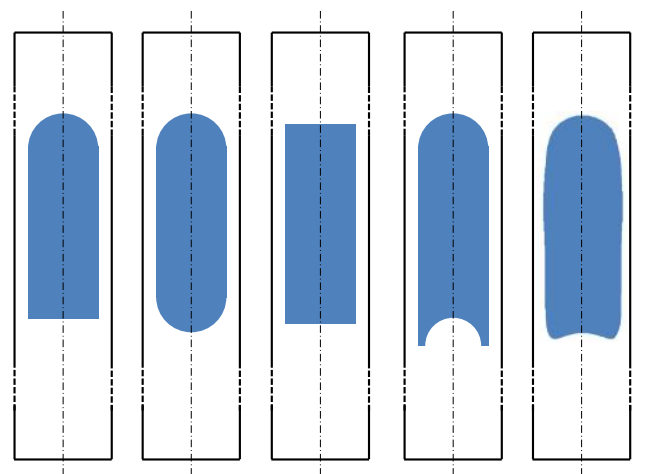

(a)

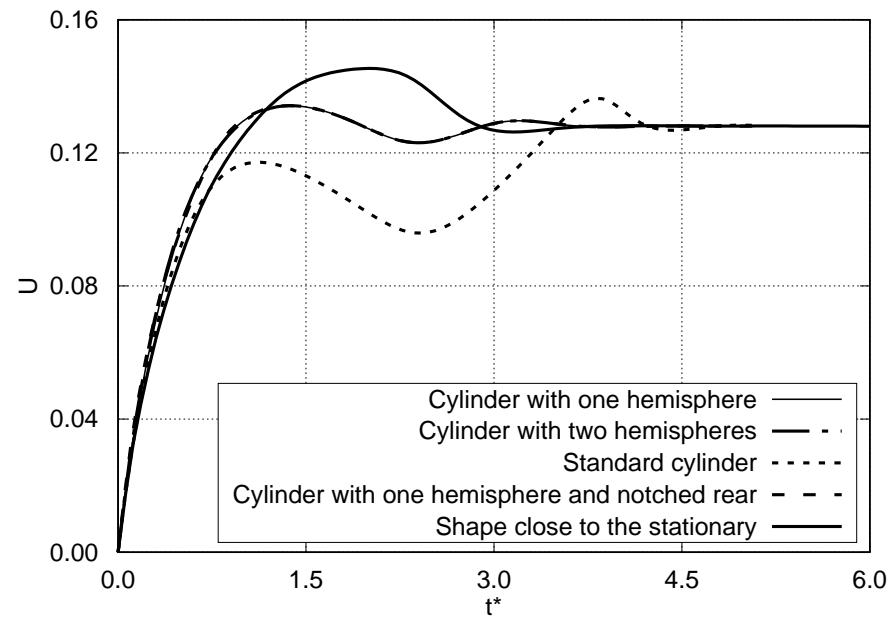

(b)

Figure 8: Sketch of (a) the different initial states tested to solve the Taylor bubble problem and (b) their velocity evolutions over dimensionless time $t^{*}$.

\subsection{Effect of the volume of the Taylor bubble}

As stated above, the initial volume of the bubble is $7.697 \cdot 10^{-6} \mathrm{~m}^{3}$, corresponding to a parameter $k$ equal to 1.29. A comparative study varying this parameter has been carried out, in order to investigate the influence of the volume of the bubble in the results.

A set of 11 cases has been run, corresponding to the following $k$ numbers: $0.4,0.5,0.6,0.7,0.8,0.9$, 1.0, 1.1, 1.2, 1.3 and 1.4. The initial shapes of the bubbles have been taken spherical when $k<2 r / D$, and cylindrical with an hemisphere at the front end when $k>2 r / D$. All other physical and geometrical parameters are the same as in previous sections. The type of mesh employed is also equivalent to the ones described above. In order to determine a suitable number of control volumes, a mesh independence study has been carried out for the extreme cases $k=0.4$ and $k=1.4$, since the geometry of the problem has changed. Tab. 5 summarized the results of this study, proving that by using a mesh with 70 control volumes per diameter, the obtained results are sufficiently precise. The dimensions of the domain are obtained as discussed above.

\begin{tabular}{cccccc}
\hline Case & $h$ & $\operatorname{Re}_{T}$ & $\Delta$ & $E_{R_{T}}$ & $E_{\Delta}$ \\
\hline \multirow{5}{*}{$k=0.4$} & $h=D / 44$ & 24.776 & -0.2175 & $6.7 \%$ & $7.5 \%$ \\
& $h=D / 58$ & 25.450 & -0.2341 & $4.2 \%$ & $0.5 \%$ \\
& $h=D / 70$ & 26.067 & -0.2362 & $1.9 \%$ & $0.4 \%$ \\
& $h=D / 84$ & 26.575 & -0.2352 & - & - \\
\hline \multirow{5}{*}{$k=1.4$} & $h=D / 44$ & 26.032 & 0.6543 & $3.6 \%$ & $5.1 \%$ \\
& $h=D / 58$ & 26.311 & 0.6629 & $2.6 \%$ & $3.7 \%$ \\
& $h=D / 70$ & 26.883 & 0.6429 & $0.5 \%$ & $1.9 \%$ \\
& $h=D / 84$ & 27.012 & 0.6307 & - & - \\
\hline
\end{tabular}

Table 5: Mesh independence study for the extreme cases $k=0.4$ and $k=1.4$, where $h$ is the characteristic cell size, $R e_{T}$ is the terminal Reynolds number, $\Delta$ is the deformation parameter, $E_{R_{T}}$ is the relative error of the terminal Reynolds number referred to the case with a denser mesh, and $E_{\Delta}$ is analogously the relative error of the deformation parameter.

Outcomes of the current study are presented in subsequent figures. On the one hand, terminal shapes 
of the bubbles are shown in Fig. 9. These forms present the following features: for $k<0.5$ the influence of the wall is not a crucial factor in the ascent of the bubble. Thus, it remains elliptical, as it corresponds to this regime for standard rising bubbles. For $k=0.6$ and $k=0.7$, the bubble tends to acquire an elongated mushroom shape, showing the transition from a standard rising bubble to a Taylor bubble, where the interaction of the bubble with the tube walls has a capital influence in the dynamics of the bubble. For $k$ greater than 0.8 and lower than 1.1, the bubble tends to take a bullet shape. Beyond this critical point, any increase in the total volume of the bubble results in increasing the axial length without changing the shape of the nose and the rear end of the bubble. Finally, for $k>1.2$ the bubble becomes slug-shaped. This behaviour is consistent with the one observed by other authors (i.e. Li et al. [49] and Amaya et al. [36]).

Additional results are shown in Fig. 10 as function of the bubble size, characterized by the parameter $k$. First, the Reynolds number is plotted in Fig. 10a For small $k$ values $(k<1)$, the velocity of the bubble grows almost linearly as $k$ does, as a result of the increase in the buoyancy force. As the bubble size becomes comparable to the tube size, the confining walls make the drag force increase. These results in a stagnation of the Reynolds number at $k \approx 1.0$, when the bubble velocity becomes independent of its volume. Furthermore, Fig. 10c shows the relationship between $k$ and the film thickness $\delta$. As the volume of the bubble increases, the film thickness is reduced until achieve a stagnation point at $k \approx 1.1$. From this point on, the value of $\delta$ stays constant and the increment of the bubble volume has an impact exclusively on the bubble axial length, but not on its proximity to the walls. This pattern is coherent with the behaviour observed in the evolution of the deformation parameter $\Delta=(L-B) /(L+B)$, where $L$ is the length of the bubble and $B$ is its width. $\Delta$ increases as $k$ increases, with a decreasing slope, due to the fact that $B$ achieves an approximately constant value for high values of $k$. Finally, the fraction of the total channel cross-sectional area occupied by the bubble $A_{\text {bubble }} / A_{\text {channel }}$ is plotted as function of $k$ in Fig. 10d. This figure reflects how large bubbles $(k>1.1)$ occupy the same fraction of the total cross-sectional area available. All these results corroborate the findings of Li et al. [49] and Amaya et al. [36, presenting similar tendencies and analogous general behaviours.

\subsection{Effect of the Eötvös number}

The importance of surface tension forces compared to body forces is measured by terms of the Eötvös number Eo. In order to quantify this influence in the Taylor bubble problem, some experiments proposed by Hayashi et al. 39] have been addressed. In this paper, several cases are studied for different sets of Eötvös and Morton numbers. For a fixed Morton number, up to four different Eötvös numbers are studied. Therefore, we chose a Morton number $M o$ of $10^{-2.5}$ and we took the following set of Eötvös numbers: $E o=\{10,33,55,77\}$.

Experimental conditions of these four cases are described below. First, density and viscosity of the suspending fluid are respectively $1220 \mathrm{~kg} / \mathrm{m}^{3}$ and $85.0 \cdot 10^{-3} \mathrm{~Pa} \cdot \mathrm{s}$. The density ratio $\eta_{\rho}$ and the viscosity ratio $\eta_{\mu}$ are respectively set to 1.26 and 0.88 . The surface tension coefficient is $0.031 \mathrm{~N} / \mathrm{m}$. Variations in the Eötvös number are obtained by changing the diameter of the tube, $D=\{0.011,0.0201,0.0261,0.0308\} \mathrm{m}$. The initial bubble has a cylindrical shape with two hemispheres, and a total volume corresponding to a parameter $k$ of 1.25. The initial radius of the bubble $r$ is calculated from Eq. 21. The used meshes are the same as the $M 2$ mesh described above, but scaled to the new sizes.

A comparison between obtained results and reference data is summarized in Tab. 6, where a good agreement can be noticed. Furthermore, Fig. 11 depicts the profiles evolution for the run cases. The final shapes represented in this figure match qualitatively well with the images of the bubble provided by Hayashi et al. [39]. As can be further seen from these results, velocity increases when the Eötvös number increases. Due to the higher buoyant forces, the elongation of the bubble also increases whit the Eötvös number. For small Eötvös numbers, the bubble tends to take an elongated egg shape (Fig. 11a and 11b). On the contrary, the 


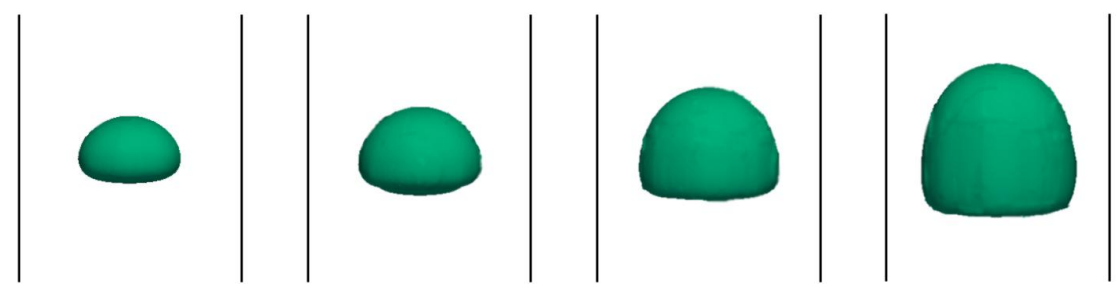

$$
k=0.4 \quad k=0.5 \quad k=0.6 \quad k=0.7
$$

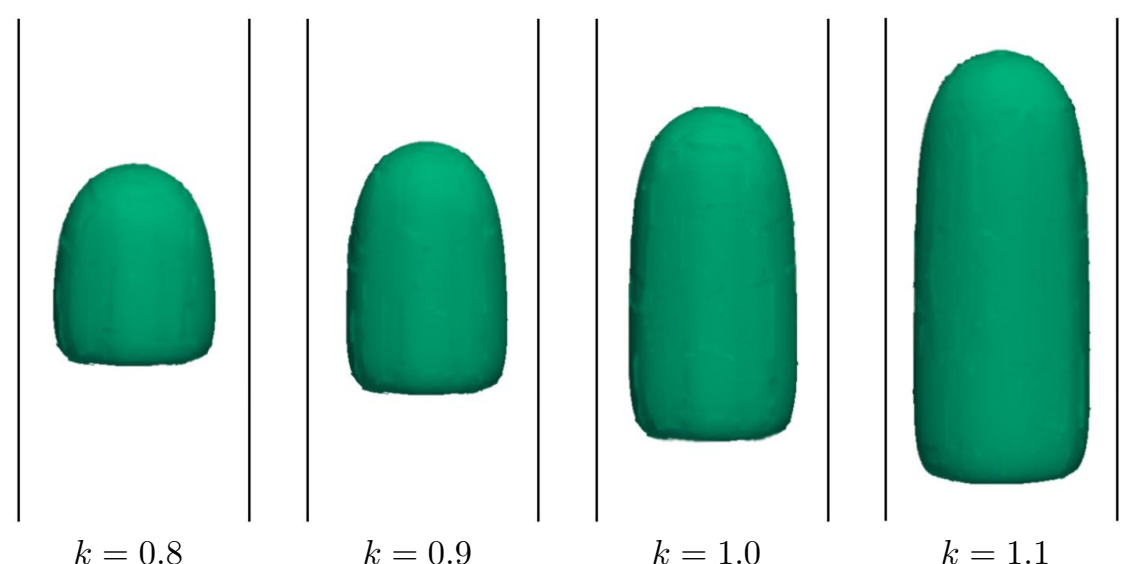

$$
k=0.8 \quad k=0.9 \quad k=1.0 \quad k=1.1
$$

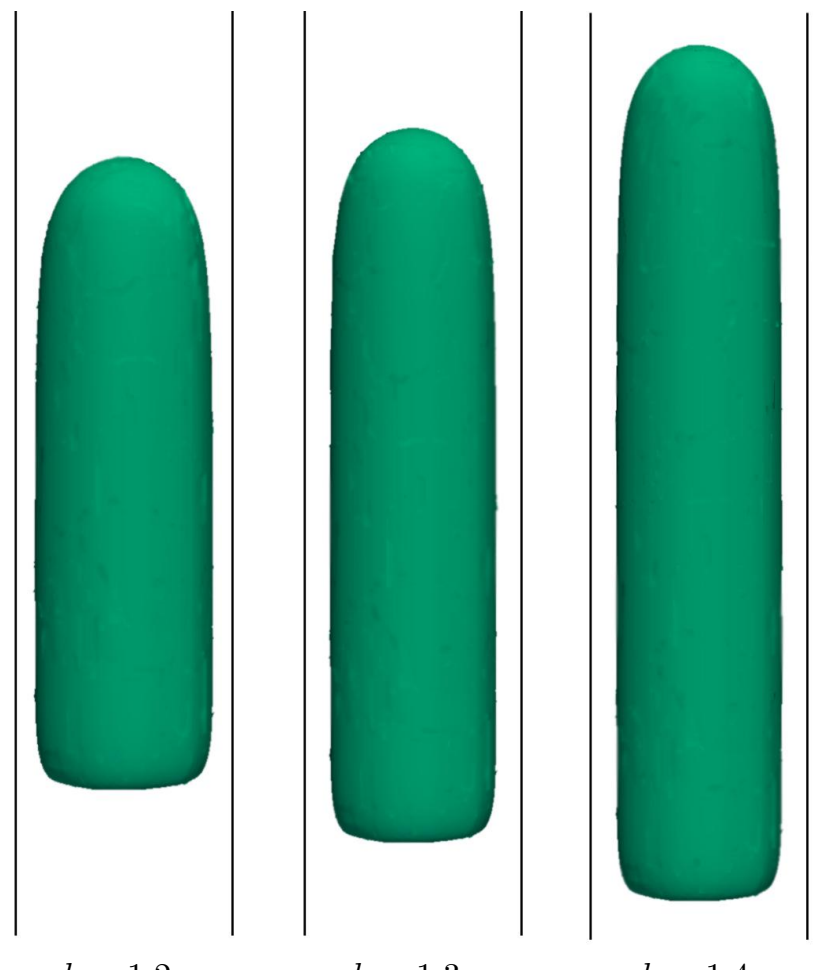

$$
k=1.2 \quad k=1.3 \quad k=1.4
$$

Figure 9: Final shapes in the study of the sensibility to the initial volume of the bubble, listed in increasing order from $k=0.4$ to $k=1.4$, with 0.1 increases. 


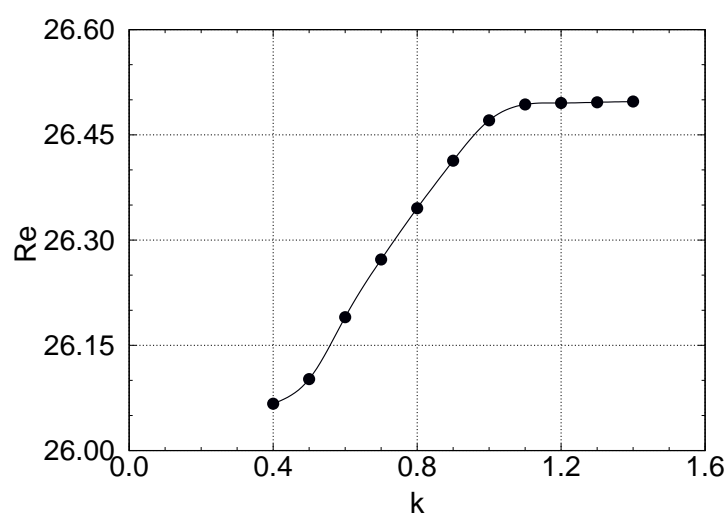

(a)

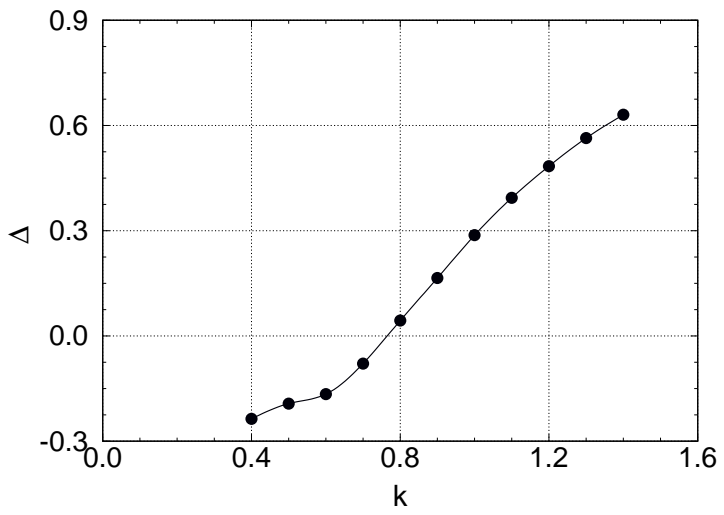

(c)

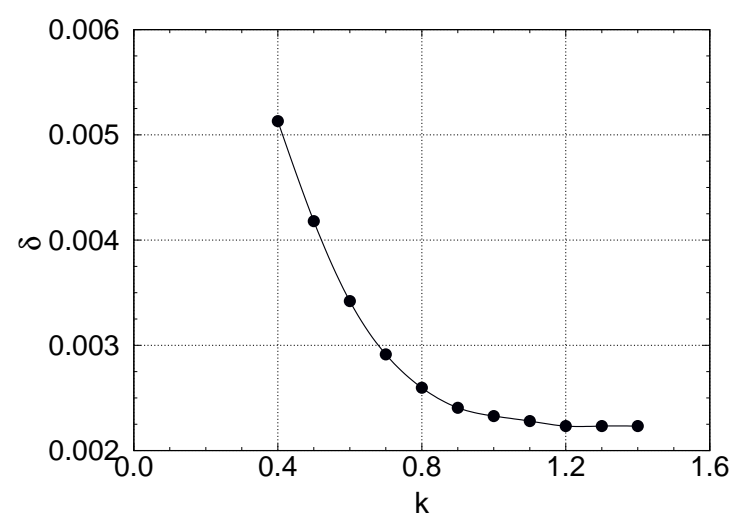

(b)

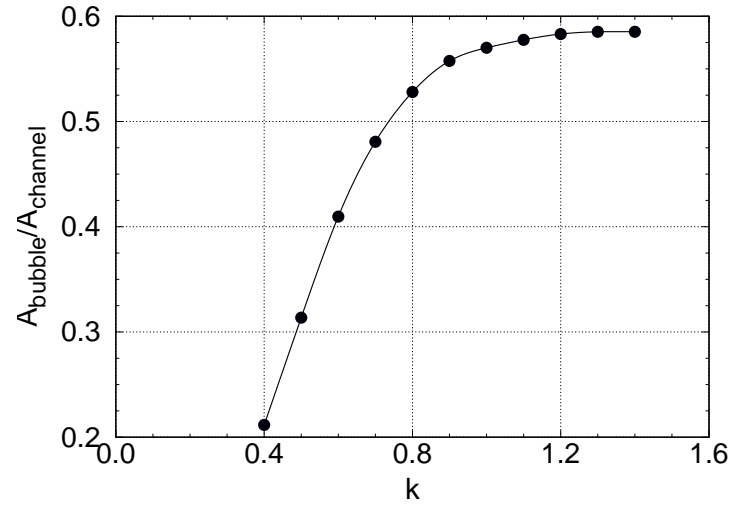

(d)

Figure 10: Comparison of (a) Reynolds number Re, (b) film thickness $\delta$, (c) deformation parameter $\Delta$ and (d) fraction of maximum channel cross-sectional area occupied by the bubble $A_{\text {bubble }} / A_{\text {channel }}$, as a function of the bubble size characterized by the parameter $k$.

bubble acquires a slug shape when higher Eötvös numbers are used (Fig. 11c and 11d).

\begin{tabular}{cccc}
\hline \multirow{2}{*}{ Eo } & \multicolumn{3}{c}{$R e_{T}$} \\
& Present work & Hayashi et al. [39] & \multirow{2}{*}{$E_{R e_{T}}$} \\
\hline 10 & 0.40 & 0.41 & $2.43 \%$ \\
33 & 8.48 & 8.7 & $2.53 \%$ \\
55 & 19.62 & 20.2 & $2.87 \%$ \\
77 & 30.57 & 31.0 & $1.39 \%$ \\
\hline
\end{tabular}

Table 6: Results comparison against the experiments of Hayashi et al. 39, where $E_{R e_{T}}$ is the relative error of the terminal Reynolds number $R e_{T}$. 


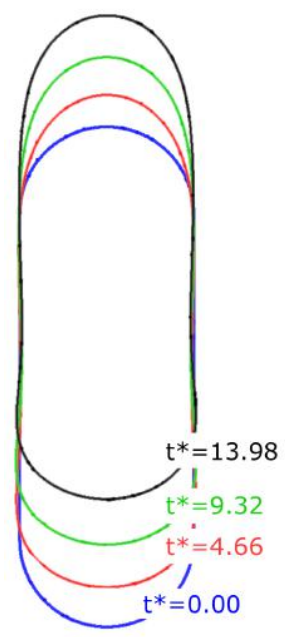

(a)

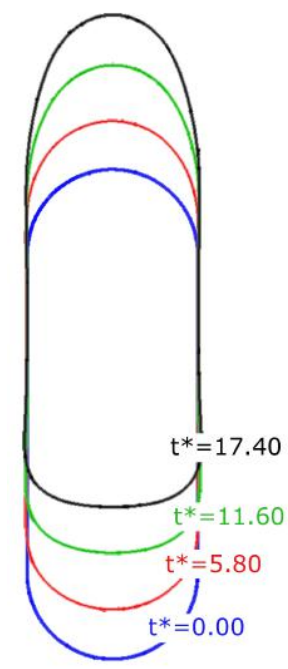

(b)

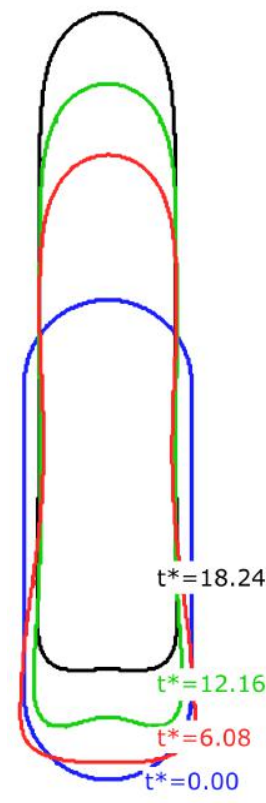

(c)

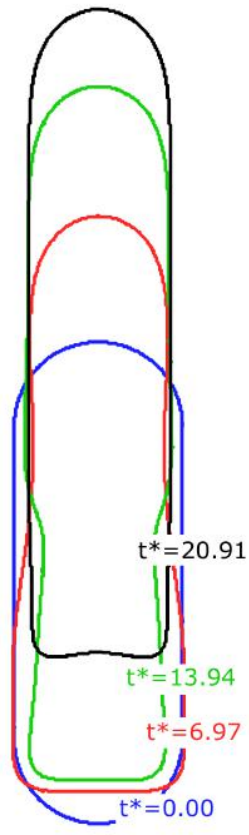

(d)

Figure 11: Evolution profiles of the three-dimensional Taylor bubble, corresponding to the different Eötvös numbers tested, i.e. (a) $E o=10$, (b) $E o=33$, (c) $E o=55$ and (d) $E o=77$. The other parameters have remained the same.

\subsection{Effect of the Morton number}

In order to study the particularities of the problem when varying the Morton number, we chose some of the cases studied by Quan [34, and we simulated them. In the mentioned paper, the inverse viscosity number $N_{f}$ is used to describe the flow regime, instead of the Morton number Mo. Both dimensionless numbers are related by the following expression:

$$
N_{f}=\left(\frac{E o^{3}}{M o}\right)^{1 / 4}
$$

On the one hand, the viscosity of the first fluid is determined from $N_{f}$, taking $g=9.85 \mathrm{~m} / \mathrm{s}^{2}$. We select the following set of inverse viscosity numbers to be tested: $N_{f}=\{291,200,109,44,22,16\}$. On the other hand, the density of the suspending fluid is $1220 \mathrm{~kg} / \mathrm{m}^{3}$. The density ratio $\eta_{\rho}$ and the viscosity ratio $\eta_{\mu}$ are both set to 100 . The surface tension coefficient is $0.06 \mathrm{~N} / \mathrm{m}$. The tube's diameter $D$ is $0.032 \mathrm{~m}$. The initial bubble has a cylindrical shape with two hemispheres, with $k=1.14$. The initial radius of the bubble $r$ is obtained by computing Eq. 21 . The used mesh is the same as the $M 2$ mesh described above, but scaled to fit the new geometry.

Tab. 7 summarizes the obtained terminal Reynolds numbers $\left(R e_{T}\right)$ compared against the ones reported by Quan [34. Good agreement was found between both sets of results. The slight differences between these results are caused by the different numerical approaches, and mainly because Quan is using an axisymmetric solver. Fig. 12 shows the profile's evolution and the final form of the tested cases. Again, the agreement of these results is qualitatively good in comparison with the ones obtained by Quan [34. When decreasing $M o$ (i.e. increasing $N_{f}$ ), some general effects are observed in the performance of the problem. First, the bubble rises faster, as a result of the decrease in the viscous force. Additionally, the slug shape is progressively transformed into a skirted oval shape. The length of the main body of the bubble decreases which indicate 
that the reduction in the viscous forces tends to compress the bubble. It becomes thicker, which is evidenced by a narrower film region between the tube wall and the bubble.

\begin{tabular}{ccccc}
\hline \multicolumn{2}{c}{ Regime } & \multicolumn{3}{c}{$\operatorname{Re}_{T}$} \\
$N_{f}$ & $M o$ & Present work & Quan $[34]$ & $E_{R e_{T}}$ \\
\hline 291 & $2.12 \cdot 10^{-3}$ & 95.97 & 97.0 & $1.06 \%$ \\
200 & $9.53 \cdot 10^{-3}$ & 65.65 & 65.6 & $0.07 \%$ \\
109 & $1.36 \cdot 10^{-1}$ & 32.78 & 33.6 & $2.44 \%$ \\
44 & 4.07 & 10.59 & 10.9 & $2.83 \%$ \\
22 & $6.31 \cdot 10^{1}$ & 3.59 & 3.8 & $5.42 \%$ \\
16 & $2.33 \cdot 10^{2}$ & 1.69 & 1.8 & $6.11 \%$ \\
\hline
\end{tabular}

Table 7: Comparison of results against the numerical experiments of Quan [34], where $E_{R e_{T}}$ is the relative error of the terminal Reynolds number $R e_{T}$.

\subsection{Effect of channel inclination}

A comprehensive study of the Taylor bubble behaviour under non-vertical inclinations is herewith presented. We chose the work of Shosho and Ryan [30] as reference. In this paper, several mixtures were experimentally tested in cylindrical inclined tubes.

The selected parameters for the study are presented below. The inside diameter of the tube $D$ is $0.0254 \mathrm{~m}$. The bubble's initial shape is a cylinder with a diameter $d$ of $0.84 D$. This cylinder is ended by two hemispheres, and it has a total length of $2 d$, corresponding to $k=1.14$. The density of the suspending fluid is $1320.02 \mathrm{~g} / \mathrm{m}^{3}$ and its viscosity $0.191 \mathrm{~Pa} \cdot \mathrm{s}$. Surface tension coefficient is set to $0.051 \mathrm{~N} / \mathrm{m}$. These parameters give rise to a Eötvös number $E o$ of 40.97 and a Morton number $M o$ of 0.0746 . The density ratio $\eta_{\rho}$ and viscosity ratio $\eta_{\mu}$ are both set to 100. It is important to point out that these ratios do not match with the actual ratio of corn syrup-air mixture used in the experiment of Shosho and Ryan [30. However, such large density and viscosity ratios give rise to numerical instabilities, and terminal velocity and bubble shapes are minimally upset when smaller ratios are used [34].

The new flow regime and the non-axisymmetric configuration of the current case, bring about a more challenging problem compared to the previous ones. Thus, a new mesh is designed to meet the resolution requirements of the current simulations. It is composed of triangular prismatic control volumes. A mesh independence study has been performed for the extreme case $(\theta=5 \pi / 12)$, in order to obtain a suitable mesh which accurately reproduce the physics of the problem. The results of this study are presented in Tab. 8, showing that a mesh with 147 control volumes per diameter seems to be a suitable mesh to study this problem.

\begin{tabular}{cccccc}
\hline Case & $h$ & $F r$ & $\Delta$ & $E_{F r}$ & $E_{\Delta}$ \\
\hline \multirow{5}{*}{$\theta=5 \pi / 12$} & $h=D / 103$ & 0.3297 & 0.7239 & $1.0 \%$ & $6.5 \%$ \\
& $h=D / 123$ & 0.3304 & 0.6585 & $0.4 \%$ & $3.1 \%$ \\
& $h=D / 147$ & 0.3307 & 0.6755 & $0.2 \%$ & $0.6 \%$ \\
& $h=D / 165$ & 0.3316 & 0.6796 & - & - \\
\hline
\end{tabular}

Table 8: Results of the mesh independence study for the extreme case $\theta=5 \pi / 12$, where $h$ is the characteristic cell size, $F r$ is the Froude number, $\Delta$ is the deformation parameter, $E_{F r}$ is the relative error of the Froude number referred to the case with denser mesh, and $E_{\Delta}$ is analogously the relative error of the deformation parameter.

The distance $h_{o}$ from the bubble rear end to the outflow should also be reconsidered. The use of Eq. 22 could a priori not be convenient for inclined cases, where their particular configurations give rise to longer 


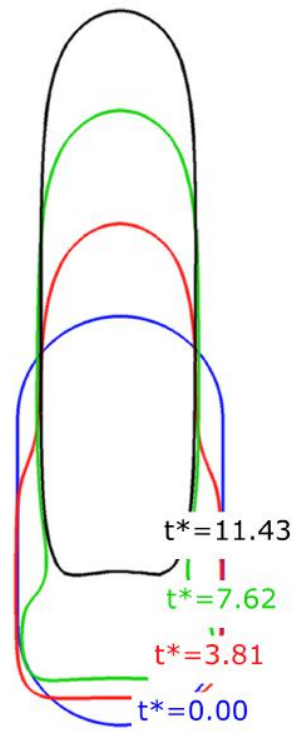

(a)

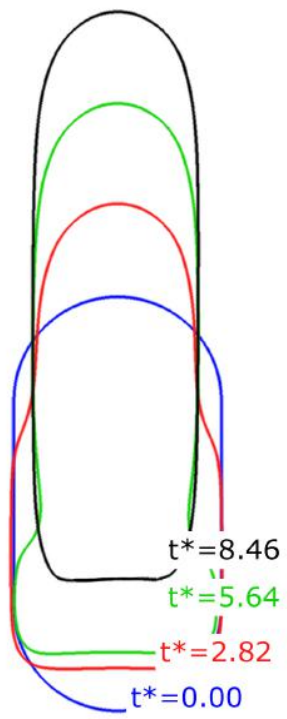

(b)

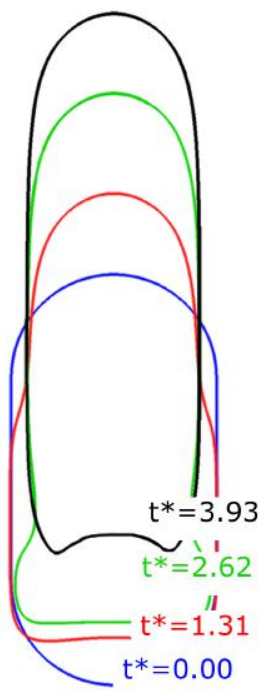

(c)

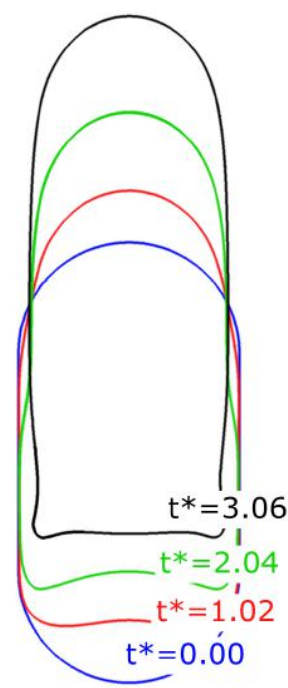

(d)

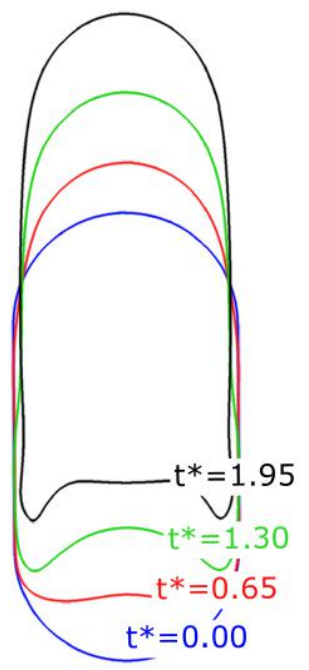

(e)

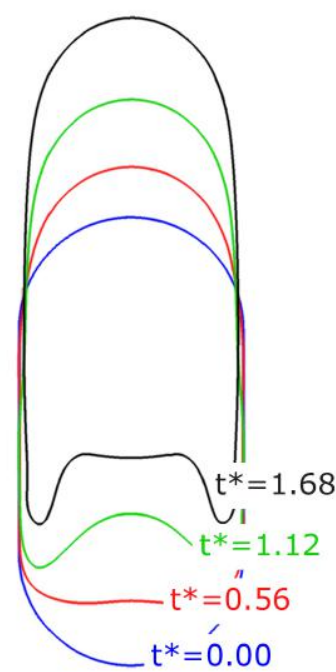

(f)

Figure 12: Evolution profiles of the three-dimensional Taylor bubble, corresponding to the different inverse viscosity numbers tested, i.e. (a) $N_{f}=16$, (b) $N_{f}=22$, (c) $N_{f}=44$, (d) $N_{f}=109$, (e) $N_{f}=200$ and (f) $N_{f}=291$.

wakes. Therefore, a particular study of the influence of the distance $h_{o}$ (controlled by the safety constant $\mathcal{C}_{h_{o}}$ ) has been conducted. Results of this study have been summarized in Tab. 9), showing that the deformation parameter and the Froude number are minimally upset by increasing $h_{o}$, when $\mathcal{C}_{h_{o}} \approx 2.0$. This fact reveals that for $\mathcal{C}_{h_{o}}=1.8$ the obtained $h_{o}$ from Eq. 22 is enough to accurately reproduce the physics of the problem.

Thus, the new mesh consists of $2.9 \cdot 10^{6}$ cells and is obtained by a constant step extrusion of a twodimensional grid along the tube axis. The total tube length $y_{\text {length }}$ is $5 D$, the number of planes in which the vertical axis is divided $N_{\text {length }}$ is 334 , the number of cells per plane is 8546 , and the characteristic cell 


\begin{tabular}{cccccc}
\hline Case & $\mathcal{C}_{h_{o}}$ & $F r$ & $\Delta$ & $E_{F r}$ & $E_{\Delta}$ \\
\hline \multirow{3}{*}{$\theta=5 \pi / 12$} & $\mathcal{C}_{h_{o}}=2.27$ & 0.3307 & 0.6755 & $0.6 \%$ & $1.0 \%$ \\
& $\mathcal{C}_{h_{o}}=2.60$ & 0.3318 & 0.6749 & $0.3 \%$ & $0.5 \%$ \\
& $\mathcal{C}_{h_{o}}=2.90$ & 0.3328 & 0.6735 & - & - \\
\hline
\end{tabular}

Table 9: Study of the influence of the distance $h_{o}$ (controlled by $\mathcal{C}_{h_{o}}$ ) for the extreme case $\theta=5 \pi / 12$, where $\mathcal{C}_{h_{o}}$ is the safety constant of Eq. 22 $F r$ is the Froude number, $\Delta$ is the deformation parameter, $E_{F r}$ is the relative error of the Froude number referred to the case with longer domain, and $E_{\Delta}$ is analogously the relative error of the deformation parameter.

dimension is $h_{\min }=2.283 \cdot 10^{-4} \mathrm{~m}$.

The inclination of the channel is controlled by setting the angle $\theta$ between the tube's axis and the gravity vector. The set of studied angles is $\theta=\{0, \pi / 6, \pi / 4, \pi / 3,5 \pi / 12\}$ (in radians).

In Tab. 10 and Fig. 13b a comparison of the results obtained in the present work against those of Shosho and Ryan [30] is presented. This comparison is made in terms of the Froude number Fr, finding a good agreement. Furthermore, Fig. 13b shows the general tendency of the Taylor bubble terminal velocity when the channel inclination increases: it rises and reaches its maximum at an inclination close to $\pi / 4$, and then decreases. This behaviour was noted by other researchers in many different configurations, i.e. with arbitrary cross-section channels [50] or with different kinds of mixtures [30. Moreover, Fig. 13a] shows the time evolution of the Froude number for the different inclination angles.

\begin{tabular}{|c|c|c|c|}
\hline \multirow{2}{*}{$\theta$} & \multicolumn{2}{|r|}{$F r$} & \multirow{2}{*}{$E_{F r}$} \\
\hline & Present work & Shosho and Ryan 30. & \\
\hline 0 & 0.2949 & 0.2854 & $3.33 \%$ \\
\hline$\pi / 6$ & 0.3504 & 0.3520 & $0.45 \%$ \\
\hline$\pi / 4$ & 0.3733 & 0.3760 & $0.72 \%$ \\
\hline$\pi / 3$ & 0.3679 & 0.3627 & $1.43 \%$ \\
\hline $5 \pi / 12$ & 0.3307 & 0.3173 & $4.22 \%$ \\
\hline
\end{tabular}

Table 10: Comparison of results against the experiments of Shosho and Ryan [30], where $F r$ is the Froude number and $E_{F r}$ is the relative error.

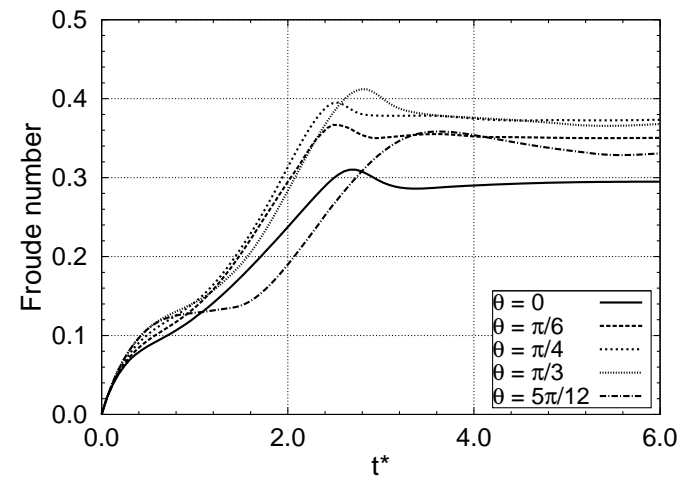

(a)

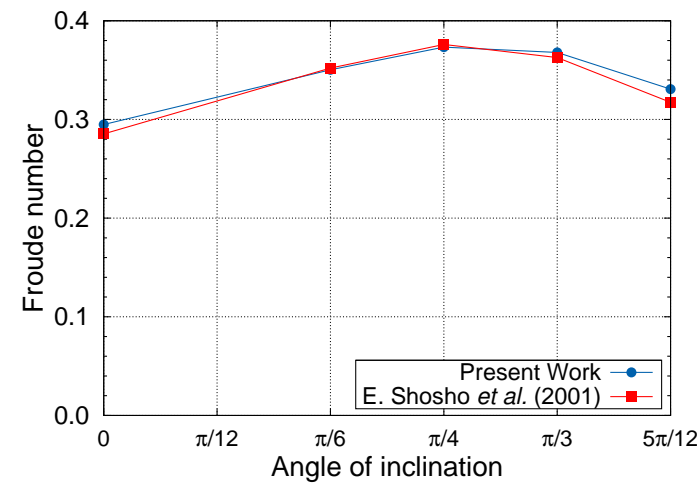

(b)

Figure 13: Sketch of (a) the evolution of the Froude number over dimensionless time $t^{*}=t g^{1 / 2} D^{-1 / 2}$ for the tested inclinations, and (b) terminal Froude number for the tested inclination angles, compared against results of Shosho and Ryan 30 . 


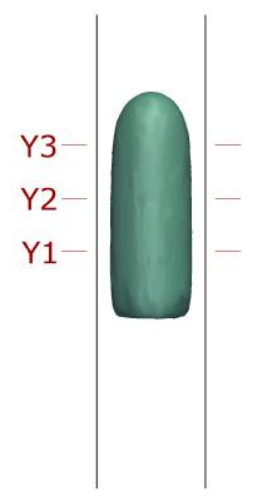

(a)

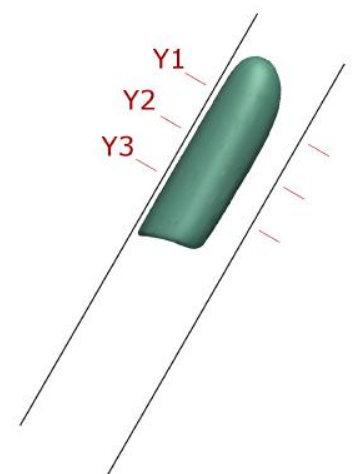

(b)

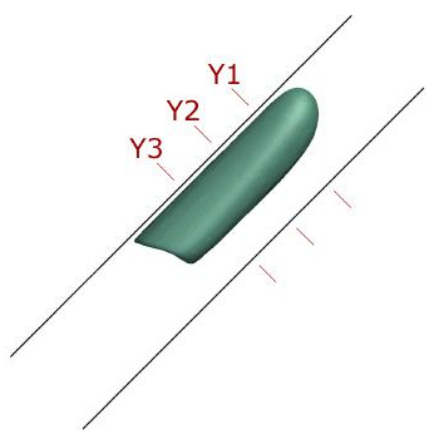

(c)

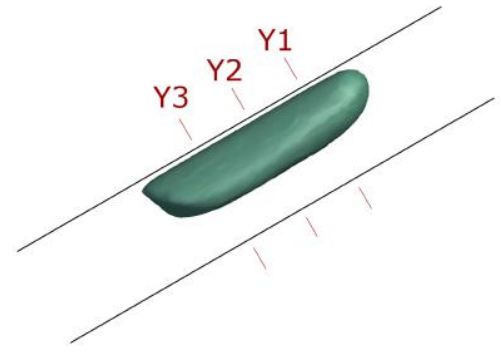

(d)

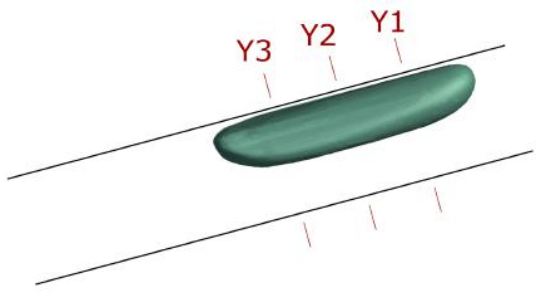

(e)

Figure 14: Three dimensional shape of the tested cases in the study of tube inclination:(a) 0 , (b) $\pi / 6$, (c) $\pi / 4$, (d) $\pi / 3$ and (e) $5 \pi / 12$. Labels $Y 1, Y 2$ and $Y 3$ mark the elevations at where streamlines and the vorticity field are analysed in Figs. 17.21

Further results are presented in the following figures. First, the final 3D bubble shapes are shown in Fig. 14. This figure shows how the average diameter of the bubble is reduced as the inclination increases, leading to a growth in the bubble length. Additionally, the distance between the bubble and the wall is reduced as the inclination angle increases. Similarly, the bubble nose gets closer to the wall as the channel is inclined. The lateral region opposite to the wall remains practically parallel to it.

Fig. 15 depicts the pressure fields of the different studied inclinations. As seen in this figure, the range of variation of the pressure is similar for the different angles. Moreover, the illustrations show how the pressure isosurfaces have a tendency of being perpendicular to the gravity vector.

Furthermore, Fig. 16 shows the streamlines for all inclination angles in the $X Y$ plane. In the vertical case (Fig. 16a), a pair of elongated counter-rotating vortices are found inside the bubble. The symmetry of this configuration is lost as the tube is inclined. The vortex at the far side of the wall is still formed, while the one close to the wall opens up reducing its size and moving downward.

Finally, Figs. 17 21 depict the streamlines and the Y-component of the vorticity field in the $X Z$ plane at three different elevations of $y$-axis. The placement of these elevations is obtained by dividing the bubble's total length into three parts. Fig. 14 shows these positions for the different inclination angles, labeled as $Y 1$, 


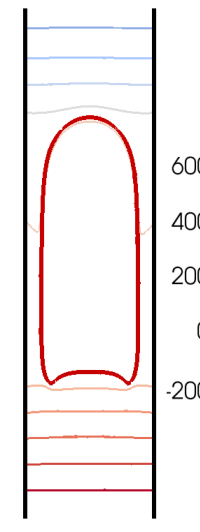

(a)

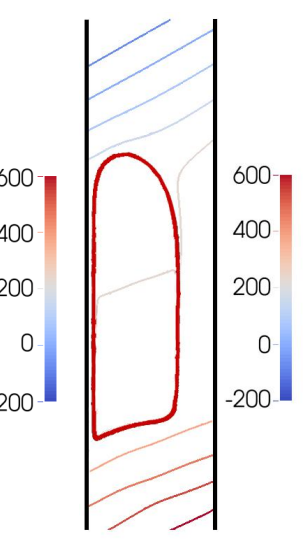

(b)

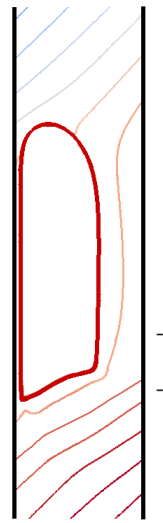

(c)

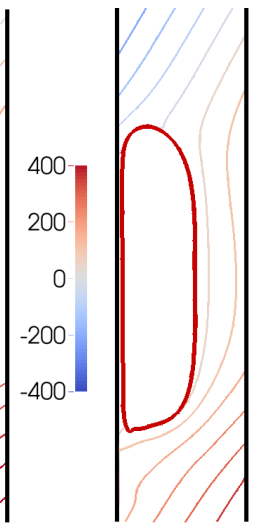

(d)

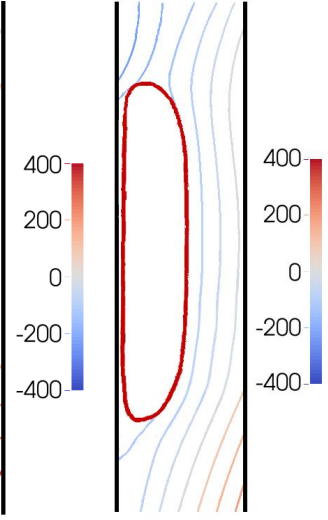

(e)

Figure 15: Pressure fields (in Pa) for all inclination angles studied: (a) 0, (b) $\pi / 6$, (c) $\pi / 4$, (d) $\pi / 3$ and (e) $5 \pi / 12$.

$Y 2$ and $Y 3$ in order of increasing height. The variation ranges of the vorticity are within the same order of magnitude in all inclination angles and all elevations, although it slightly increases with the inclination angle. For the vertical case (Fig. 17), the streamlines present a well-defined configuration of counter-rotating vortices along the bubble perimeter. This axis-symmetric arrangement is vanished as the inclination angle increases. In general, for the inclined cases (Figs. 18 21), two regions of opposite vorticity can be found in both sides of the bubble. This configuration becomes increasingly clear when the inclination rises. The lowest elevation $Y 1$ seems more perturbed and asymmetric due to the effect of the instability of the rear end. Secondary vortices appear at the perimeter of the tube in some specific configurations (e.g. see Fig. 20a). The $Y 3$ plot seems similar for the different inclination angles (see Figs. 18c, 19c, 20c and 21c), presenting an asymptotic-like pattern starting at the right side of the tube and going to the centre of the bubble. This behaviour is consistent with results reported by 36 .

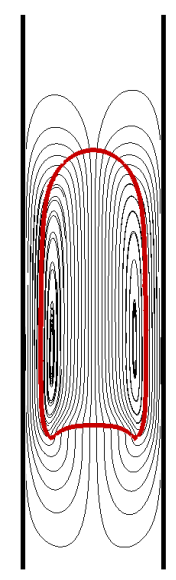

(a)

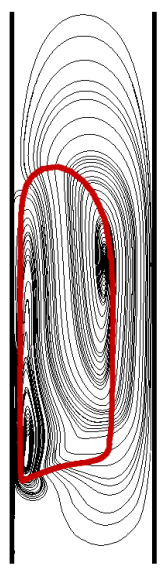

(b)

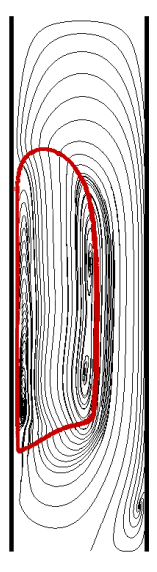

(c)

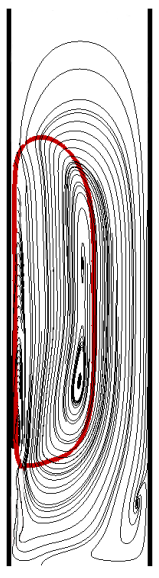

(d)

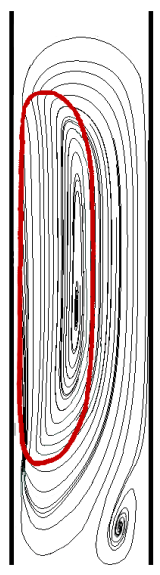

(e)

Figure 16: Streamlines in $X Y$ plane for all inclination angles studied: (a) 0, (b) $\pi / 6$, (c) $\pi / 4$, (d) $\pi / 3$ and (e) $5 \pi / 12$. 

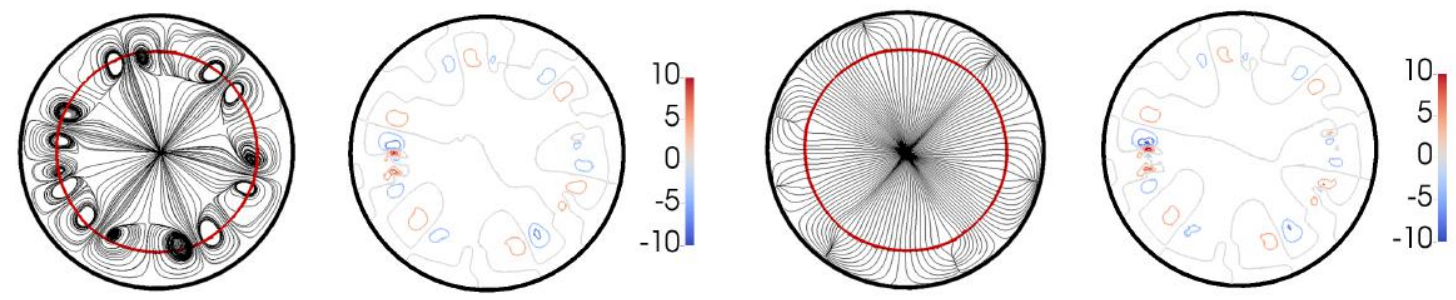

(a)

(b)
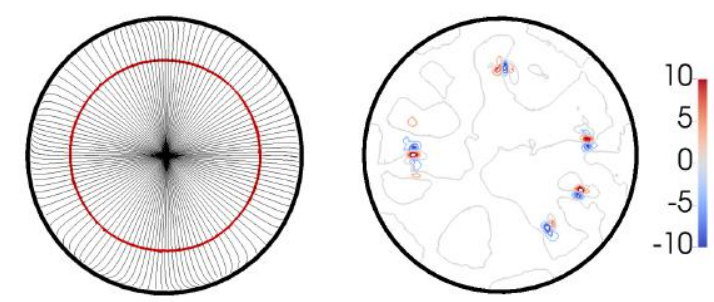

(c)

Figure 17: Streamlines and vorticity field $\left(\mathrm{s}^{-1}\right)$ in $X Z$ plane at the three different elevations (a) $Y 1$, (b) $Y 2$ and (c) $Y 3$, corresponding to the 0 inclination case.
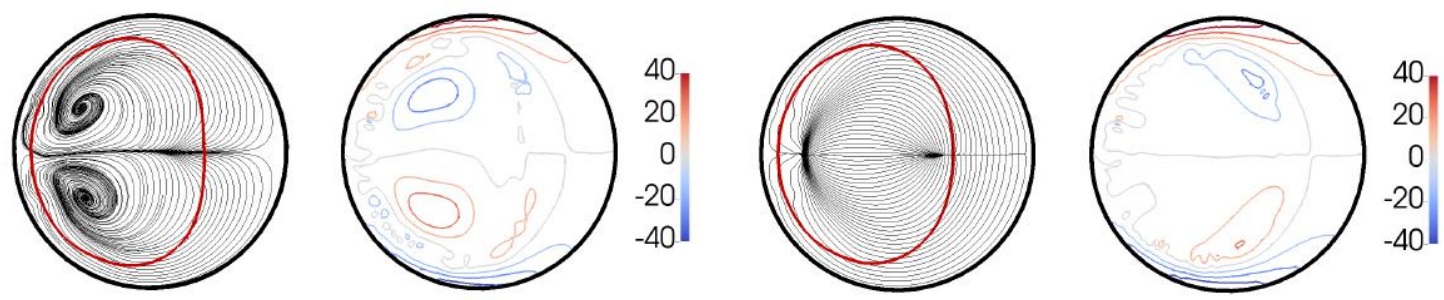

(a)
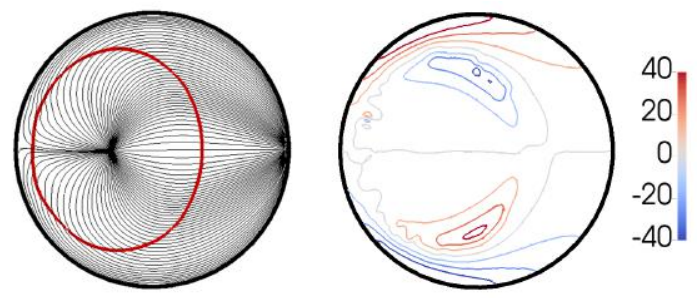

(c)

Figure 18: Streamlines and vorticity field $\left(\mathrm{s}^{-1}\right)$ in $X Z$ plane at the three different elevations (a) $Y 1$, (b) $Y 2$ and (c) $Y 3$, corresponding to the $\pi / 6$ inclination case. 


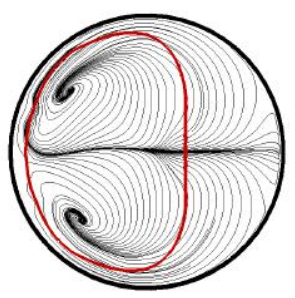

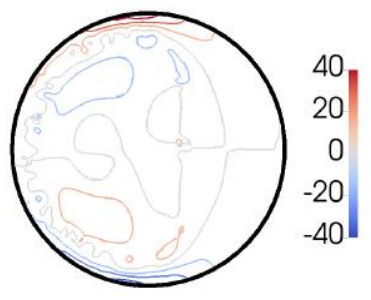

(a)
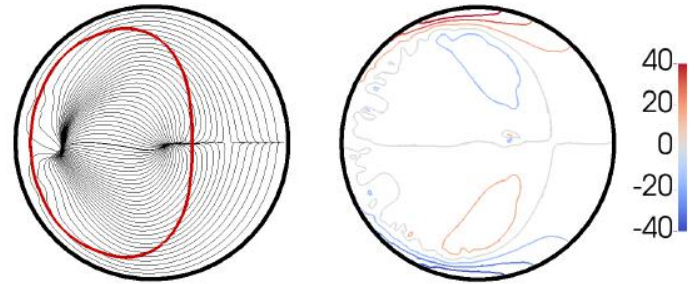

(b)
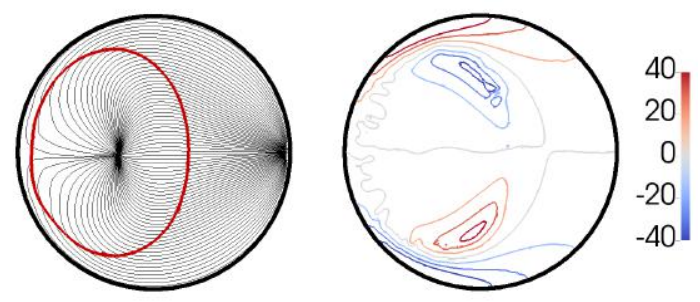

(c)

Figure 19: Streamlines and vorticity field $\left(\mathrm{s}^{-1}\right)$ in $X Z$ plane at the three different elevations (a) $Y 1$, (b) $Y 2$ and (c) $Y 3$, corresponding to the $\pi / 4$ inclination case.

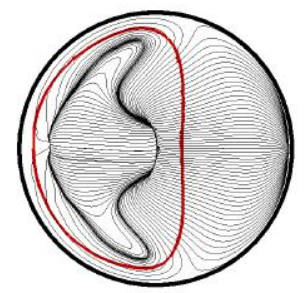

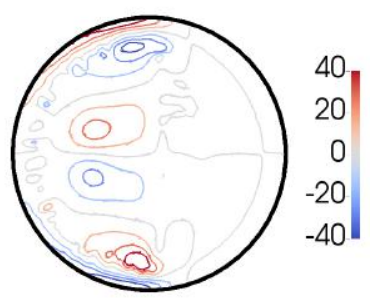

(a)
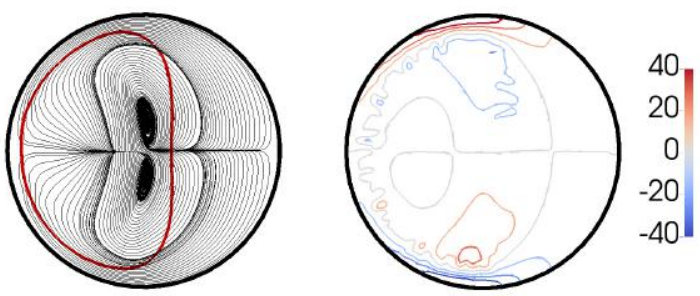

(b)
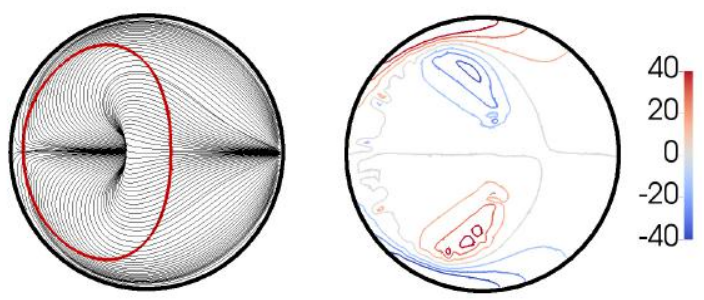

(c)

Figure 20: Streamlines and vorticity field $\left(\mathrm{s}^{-1}\right)$ in $X Z$ plane at the three different elevations (a) $Y 1$, (b) $Y 2$ and (c) $Y 3$, corresponding to the $\pi / 3$ inclination case.

\section{Conclusions}

In the present work, an Arbitrary Lagrangian-Eulerian approach to optimize the computational domain in Taylor bubbles problems have been posed, within a Conservative Level Set framework. The method was used to perform a deep study of the Taylor bubble problem.

By using an optimized domain, the efficiency of the simulation can be notably improved, due to the fact that it is no longer necessary to solve regions far from the vicinity of the bubble, which are not of interest 


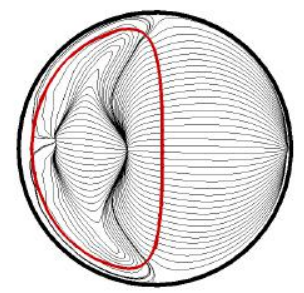

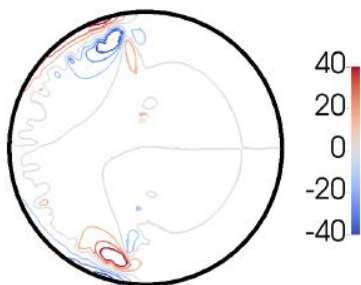

(a)
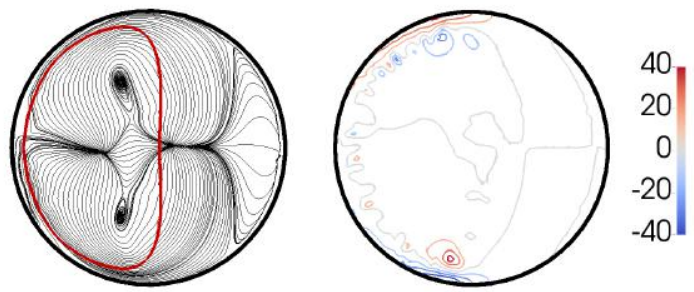

(b)
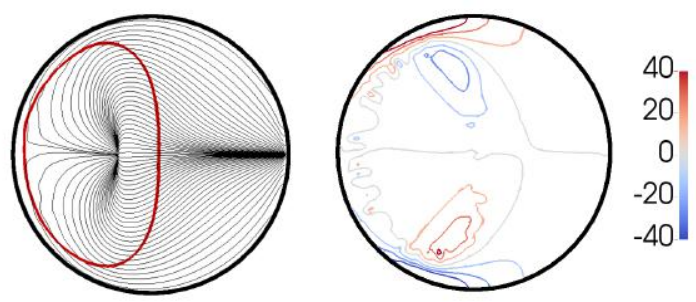

(c)

Figure 21: Streamlines and vorticity field $\left(\mathrm{s}^{-1}\right)$ in $X Z$ plane at the three different elevations (a) $Y 1$, (b) $Y 2$ and (c) $Y 3$, corresponding to the $5 \pi / 12$ inclination case.

in rising bubble problems. The method is based on a moving grid that follows the ascent of the buoyant bubble. It showed a proper numerical stability and a good performance. The only noteworthy disadvantage of this method is that it needs using open boundaries (namely, inflow and outflow), which usually requires a careful numerical treatment, as explained in Sec. 3. In effect, the formulation and the placement of the open boundaries (specially the outflow) are burdensome factors to be taken into account in designing these boundaries. Some progresses have been made in this regard: first, a new outflow boundary condition has been developed, that aims to minimize the global mass error; second, an enhanced mass correction step has been proposed, which improves the numerical stability of the overall algorithm; and third, practical considerations about the placement of those boundaries have been addressed, with the goal of optimizing the domain dimensions and being able to minimize the computational cost of the simulations.

The previous method was tested in the three-dimensional standard rising bubble. Accurate results were obtained, proving the validity of this method to optimize the computational domain in bubbles problems. We then tackled the challenging problem of the three dimensional Taylor bubble. To the best knowledge of the authors, this problem has not been addressed by using a CLS method. A full study was carried out, including the sensitivity analyses with respect to the initial shape of the bubble, the initial volume of the bubble, the flow regime and the inclination of the channel. All the aforementioned tests have been contrasted with both experimental and numerical studies available in the literature, finding a good agreement.

\section{Appendix A Calculations of bubble properties}

For the sake of results comparison, and also to carry out some calculations, different kinematic properties of the bubble are needed. First, the bubble centre of mass is calculated as:

$$
\mathbf{x}_{\text {bubble }}=\frac{\sum_{n} \mathbf{x}_{n} \phi_{n} V_{n}}{\sum_{n} \phi_{n} V_{n}}
$$


where subscript $n$ denotes that the corresponding variable is evaluated at the node $n$ under consideration. Second, bubble velocity is given by:

$$
\mathbf{v}_{\text {bubble }}=\frac{\sum_{n} \mathbf{v}_{n} \phi_{n} V_{n}}{\sum_{n} \phi_{n} V_{n}}
$$

Finally, the sphericity $\zeta_{3 D}$ measures the deformation with respect to a perfect spherical bubble. It is defined as:

$$
\zeta_{3 D}=\frac{4 \pi r^{2}}{\sum_{n}\left\|\nabla \phi_{n}\right\| V_{n}}
$$

where $r$ is the bubble initial radius.

\section{Appendix B Mass correction}

As explained in Sec. 3.4, the outflow boundary condition requires a mass correction step which is performed after calculating the predictor velocity (see Sec. 3). In the literature this is usually done by adding the corrective mass $m^{\text {cor }}$ to the mass flow $m$ of the corresponding outflow boundary node [42]:

$$
m_{b}^{\text {cor }}=\sum_{b f} m_{b} \frac{A_{b}}{\sum_{o b f} A}
$$

where $A$ is the face area. Subscript $b$ denotes that the corresponding variable is evaluated at the boundary node under consideration. Summation $\sum_{b f} m_{b}$ is performed over all boundary faces in the entire domain and summation $\sum_{o b f} A$ is performed just over the outflow boundary faces. This method for mass correction might have the drawback of the formation of large local mass errors in the corner cells of the domain due to the physical characteristics of the problem, causing numerical instabilities. In order to avoid this issue, a modified mass correction step is proposed.

In this way, the global mass balance error $\Delta m$ is distributed on a weighted basis over the outflow boundary nodes. A weighting coefficient $w$ is assigned to each node of the outflow boundaries. Thus, the nodes near the corners have small coefficients, and the central nodes have larger ones. Those requirements are accomplished by the well-known Gaussian function [51, whose expression over a generic surface can be given by:

$$
w_{b}(\alpha, \beta)=\frac{\mathbf{v}_{\text {bubble }} \cdot \mathbf{n}}{\left\|\mathbf{v}_{\text {bubble }}\right\|\|\mathbf{n}\|} \exp \left\{-\frac{1}{2} \chi\left[\left(\alpha-\frac{S_{\alpha}}{2}\right)^{2}+\left(\beta-\frac{S_{\beta}}{2}\right)^{2}\right]\right\}
$$

where $\mathbf{n}$ is the unit normal vector, $\chi$ is a shape control parameter of the weight function $(\chi \approx 1), \alpha$ and $\beta$ are curvilinear coordinates over the boundary surface, and $S_{\alpha}$ and $S_{\beta}$ are projections of the surface in $\alpha$ and $\beta$ directions, respectively.

\section{Acknowledgments}

This work has been financially supported by the Ministerio de Economía y Competitividad, Secretaría de Estado de Investigación, Desarrollo e Innovación of Spain (Project ENE-2018-70672). Calculations have been performed on the JFF cluster and on Barcelona Supercomputing Center facilities (applications RESFI-2016-1-0024 and RES-FI-2016-3-0037). The authors thankfully acknowledge these institutions. 


\section{References}

[1] W. Salman, A. Gavriilidis, P. Angeli, On the formation of Taylor bubbles in small tubes, Chem. Eng. Sci. 61 (2006) 6653-6666.

[2] E. W. Llewellin, E. Del Bello, J. Taddeucci, P. Scarlato, S. J. Lane, The thickness of the falling film of liquid around a Taylor bubble, Proc. R. Soc. A. 468 (2012) 1041-1064.

[3] D. Gaudlitz, N. Adams, Numerical investigation of rising bubble wake and shape variations, Phys. Fluids. $21(2009) 1-9$.

[4] P. Schlatter, N. Adams, L. Kleiser, A windowing method for periodic inflow/outflow boundary treatment of non-periodic flows, J. Comput. Phys. 206 (2005) 505-535.

[5] J. Nordström, N. Nordin, D. Henningson, The fringe region technique and the Fourier method used in the Direct Numerical Simulation of spatially evolving viscous flows, J. Sci. Comput. 20 (1999) 1365-1393.

[6] O. Estruch, O. Lehmkuhl, R. Borrell, C. D. Pérez Segarra, A. Oliva, A parallel radial basis function interpolation method for unstructured dynamic meshes, Comput. Fluids. 80 (2013) 44-54.

[7] J.T. Batina, Unsteady Euler airfoil solutions using unstructured dynamic meshes, AIAA J. 28 (1990) 1381-1388.

[8] Y. Bazilevs, K. Takizawa, T.E. Tezduyar, Computational Fluid-Structure Interaction, Wiley, Hoboken, 2013.

[9] H. Marschall, S. Boden, C. Lehrenfeld, D. Falconi, J. Carlos, U. Hampel, A. Reusken, M. Wörner, D. Bothe, Validation of Interface Capturing and Tracking Techniques with different Surface Tension Treatments against a Taylor Bubble Benchmark Problem, Comput. Fluids. 102 (2013) 1-21.

[10] H. Rusche, Computational Fluid Dynamics of Dispersed Two-Phase Flows at High Phase Fractions, $\mathrm{PhD}$ thesis, 2002.

[11] M.C. Ruzicka, On bubbles rising in line, Int. J. Multiph. Flow. 26 (2000) 1141-1181.

[12] M. Ohta, M. Sussman, The buoyancy-driven motion of a single skirted bubble or drop rising through a viscous liquid, Phys. Fluids. 24 (2012).

[13] M. Ohta, S. Yamaguchi, Y. Yoshida, M. Sussman, The sensitivity of drop motion due to the density and viscosity ratio, Phys. Fluids. 22 (2010) 1-11.

[14] A.J. Chorin. Numerical solution of the Navier-Stokes equations, Math. Comp. 22 (1968), 745-762.

[15] J.L. Guermond, P. Minev, J. Shen, An overview of projection methods for incompressible flows, Comput. Methods Appl. Mech. Eng. 195 (2006) 6011-6045.

[16] G. Tryggvason, B. Bunner, A. Esmaeeli, D. Juric, N. Al-Rawahi, W. Tauber, J. Han, S. Nas, Y.J. Jan, A Front-Tracking Method for the Computations of Multiphase Flow, J. Comput. Phys. 169 (2001) 708-759.

[17] E. Olsson and G. Kreiss, A conservative level set method for two phase flow, J. Comput. Phys. 210 (2005) 225-246 
[18] R. Scardovelli, S. Zaleski, Direct numerical simulation of free surface and interfacial flow, Annu. Rev. Fluid Mech. (1999), 31, 567-603.

[19] M. Sussman, E. Fatemi, An efficient interface-preserving level set redistancing algorithm and its application to interfacial incompressible fluid flow, SIAMJ. Sci. Comput. (1999), 20, 1165-1191.

[20] N. Balcázar, O. Lehmkuhl, L. Jofre, J. Rigola and A. Oliva, A coupled volume-of-fluid/level-set method for simulation of two-phase flows on unstructured meshes Computers and Fluids (2016), 124, 12-19.

[21] N. Balcázar, L. Jofre, O. Lehmkuhl, J. Castro, J. Rigola, A finite-volume/level-set method for simulating two-phase flows on unstructured grids, Int. J. Multiph. Flow. 64 (2014) 55-72.

[22] N. Balcázar, O. Lehmkuhl, J. Rigola, A. Oliva, A multiple marker level-set method for simulation of deformable fluid particles, Int. J. Multiph. Flow. 74 (2015) 125-142.

[23] N. Balcázar, O. Lehmkuhl, L. Jofre, A. Oliva, Level-set simulations of buoyancy-driven motion of single and multiple bubbles, Int. J. Heat Fluid Flow. 56 (2015) 91-107.

[24] N. Balcázar, J. Rigola, J. Castro, A. Oliva, A level-set model for thermocapillary motion of deformable fluid particles, Int. J. Heat Fluid Flow. October 2016.

[25] R.M. Davies, G. Taylor, The Mechanics of Large Bubbles Rising through Extended Liquids and through Liquids in Tubes, Proc. R. Soc. A Math. Phys. Eng. Sci. 200 (1950) 375-390.

[26] E.T. White, R.H. Beardmore, The velocity of rise of single cylindrical air bubbles through liquids contained in vertical tubes, Chem. Eng. Sci. 17 (1962) 351-361.

[27] R.Sh. Abiev, I.V. Lavretsov, Hydrodynamics of gas liquid Taylor flow and liquid solid mass transfer in mini channels: Theory and experiment, Chem. Eng. J. 176-177 (2011) 57-64.

[28] S. Bhusan, S. Ghosh, G. Das, P.K. Das, Rise of Taylor bubbles through narrow rectangular channels, Chem. Eng. J. 155 (2009) 326-332.

[29] J.D. Bugg, G.A. Saad, The velocity field around a Taylor bubble rising in a stagnant viscous fluid: Numerical and experimental results, Int. J. Multiph. Flow. 28 (2002) 791-803.

[30] C.E. Shosho and M.E. Ryan, An experimental study of the motion of long bubbles in inclined tubes, Chem. Eng. Sci. 56 (1999) 2191-2204.

[31] Y.B. Zudin, Analytical solution of the problem of the rise of a Taylor bubble, Phys. Fluids. 25 (2013).

[32] T. Funada, D.D. Joseph, T. Maehara, S. Yamashita, Ellipsoidal model of the rise of a Taylor bubble in a round tube, Int. J. Multiph. Flow. 31 (2005) 473-491.

[33] M. Dang, J. Yue, G. Chen, Q. Yuan, Formation characteristics of Taylor bubbles in a microchannel with a converging shape mixing junction, Chem. Eng. J. 223 (2013) 99-109.

[34] S. Quan, Co-current flow effects on a rising Taylor bubble, Int. J. Multiph. Flow. 37 (2011) 888-897.

[35] A. Kuzmin, M. Januszewski, D. Eskin, F. Mostow, J. J. Derksen, Lattice Boltzmann study of mass transfer for two-dimensional Bretherton/Taylor bubble train flow, Chem. Eng. J. 225 (2013) 580-596.

[36] L. Amaya-Bower, T. Lee, Numerical simulation of single bubble rising in vertical and inclined square channel using lattice Boltzmann method, Chem. Eng. Sci. 66 (2011) 935-952. 
[37] R.Sh. Abiev, Bubbles velocity, Taylor circulation rate and mass transfer model for slug flow in milli- and microchannels, Chem. Eng. J. 227 (2013) 66-79.

[38] N.V. Ndinisa, D.E. Wiley, D.F. Fletcher, Computational Fluid Dynamics Simulations of Taylor Bubbles in Tubular Membranes: Model Validation and Application to Laminar Flow Systems, Chem. Eng. Res. Des. 83 (2005) 40-49.

[39] K. Hayashi, R. Kurimoto, A. Tomiyama, Terminal velocity of a Taylor drop in a vertical pipe, Int. J. Multiph. Flow. 37 (2011) 241-251.

[40] A. Harten, The artificial compression method for computation of shocks and contact discontinuities. III - Self-adjusting hybrid schemes, Math. Comp. 32 (1978) 363-389.

[41] J.U. Brackbill, D.B. Kothe, C. Zemach, A continuum method for modeling surface tension, J. Comput. Phys. 100 (1992) 335-354.

[42] A. Sohankar, C. Norberg, L. Davidson, Low-Reynolds-number flow around a square cylinder at incidence: Study of blockage, onset of vortex shedding and outlet boundary condition, Int. J. Numer. Meth. Fluids. 26 (1996) 39-56.

[43] J.H. Ferziger, M. Peric, Computational Methods for Fluid Dynamics, Springer, Berlin, 2002.

[44] R.W. Davis, E.F. Moore, A numerical study of vortex shedding from rectangles, J. Fluid Mech. 116 (1982) 475-506.

[45] S. Gottlieb and C. Shu, Total variation diminishing Runge-Kutta schemes, Math. Comp. (1998), 67, 73-85.

[46] T.Z. Harmathy, Velocity of large drops and bubbles in media of infinite or restricted extent, AIChE J. 6 (1960) 281-288.

[47] J.G. Hnat and J.D. Buckmaster, Spherical cap bubbles and skirt formation, Phys. Fluids. 19 (1976), 182-194.

[48] R. A. S. Brown, The mechanics of large gas bubbles in tubes: I. Bubble velocities in stagnant liquids, Can. J. Chem. Eng. 43 (1965) 217-223.

[49] J. Li, V. Bulusu, N.R. Gupts, Buoyancy-driven motion of bubbles in square channels, Chem. Eng. Sci. 63 (2008) 3766-3774.

[50] C. Clanet, P. Héraud, G. Searby, On the motion of bubbles in vertical tubes of arbitrary cross-sections: some complements to the Dumitrescu-Taylor problem, J. Fluid Mech. 519 (2004) 359-376.

[51] X. Pennec, Intrinsic Statistics on Riemannian Manifolds: Basic Tools for Geometric Measurements, J. Math. Imaging. Vis. 25 (2006), 127. 